\title{
Thalamic projections sustain prefrontal activity during working memory maintenance
}

\author{
Scott S. Bolkan ${ }^{1}$, Joseph M. Stujenske ${ }^{1}$, Sebastien Parnaudeau ${ }^{2}$, Timothy J. Spellman ${ }^{3}$, \\ Caroline Rauffenbart ${ }^{4,5,7}$, Atheir I. Abbas ${ }^{4,6}$, Alexander Z. Harris ${ }^{4,6}$, Joshua A. Gordon ${ }^{4,6, *}$, \\ and Christoph Kellendonk ${ }^{4,5,7,}{ }^{*}$ \\ ${ }^{1}$ Graduate Program in Neurobiology and Behavior, Columbia University, College of Physicians \\ and Surgeons, New York, New York, USA
}

2Sorbonne Universités, UPMC Paris 06, Institut de Biologie Paris Seine, UM119. Neuroscience Paris Seine, CNRS UMR8246, INSERM U1130, Paris, France

${ }^{3}$ Feil Family Brain and Mind Research Institute, Weill Cornell Medical College, New York, New York 10021, USA

${ }^{4}$ Department of Psychiatry, Columbia University, College of Physicians and Surgeons, New York, New York, USA

${ }^{5}$ Department of Pharmacology, Columbia University, College of Physicians and Surgeons, New York, New York, USA

${ }^{6}$ Division of Integrative Neuroscience, New York State Psychiatric Institute, New York, New York, USA

${ }^{7}$ Division of Molecular Therapeutics, New York State Psychiatric Institute, New York, New York, USA

\section{Abstract}

The mediodorsal thalamus (MD) shares reciprocal connectivity with the prefrontal cortex (PFC) and decreased MD-PFC connectivity is observed in schizophrenia patients. Patients also display cognitive deficits including impairments in working memory, but a mechanistic link between thalamo-prefrontal circuit function and working memory is missing. Here, using pathway-specific inhibition we found directional interactions between MD and medial PFC (mPFC), with MD-to-

\footnotetext{
Users may view, print, copy, and download text and data-mine the content in such documents, for the purposes of academic research, subject always to the full Conditions of use: http://www.nature.com/authors/editorial_policies/license.html\#terms

*Correspondence should be addressed to J.A.G. (joshua.gordon@ nih.gov) or C.K. (ck491@ cumc.columbia.edu). AUTHOR CONTRIBUTIONS

S.S.B., J.A.G. and C.K. designed the experiments. S.S.B. performed the experiments and analyzed the data. J.M.S., S.P., T.J.S., C.R., A.I.A. and A.Z.H. assisted the design, performance, analysis, and interpretation of experiments. S.S.B., J.A.G. and C.K. interpreted the results and wrote the paper.

COMPETING FINANCIAL INTERESTS

The authors declare no competing financial interests.

Publisher's Disclaimer: DISCLAIMER

This article was prepared while J.A.G. was employed at the Department of Psychiatry at Columbia University and NYSPI. The opinions expressed in this article are the author's own and do not reflect the view of the National Institutes of Health, the Department of Health and Human Services, or the United States government.
} 
mPFC supporting working memory maintenance and mPFC-to-MD supporting subsequent choice. We further identify mPFC neurons that display elevated spiking during the delay, a feature that was absent on error trials and required MD inputs for sustained maintenance. Strikingly, delaytuned neurons had minimal overlap with spatially-tuned neurons and each mPFC population exhibited mutually exclusive dependence on MD and hippocampal inputs. These findings indicate a role for the MD in sustaining prefrontal activity during working memory maintenance. Consistent with this idea we found that enhancing MD excitability was sufficient to enhance task performance.

\section{INTRODUCTION}

The prefrontal cortex (PFC) is a locus for higher-order cognition and executive control across species ${ }^{1}$. In several mental disorders such as schizophrenia, PFC dysfunction is observed in concert with a variety of cognitive impairments, including deficits in working memory ${ }^{2-4}$. However, there is growing appreciation that PFC function cannot be divorced from that of its densely interconnected thalamic partners, such as the mediodorsal thalamus $(\mathrm{MD})^{5-7}$. Indeed, not only does the PFC share dense reciprocal connectivity with the $\mathrm{MD}^{8}$, manipulations of MD function in animals produce cognitive impairments reminiscent of PFC dysfunction ${ }^{9-12}$. Brain imaging studies have also shown MD dysfunction in patients with schizophrenia ${ }^{13,14}$, with increasing evidence for decreased functional connectivity between the thalamus and PFC ${ }^{15-17}$. In spite of these findings, a circuit level understanding of how thalamo-prefontal circuits engage in cognition is lacking. Such an understanding will be essential to elucidating how circuit alterations contribute to cognitive dysfunction in disorders such as schizophrenia.

To address this issue we investigated thalamo-prefrontal interactions during a spatial working memory task in which mice had to choose a spatial location that differed from one they had sampled prior to a brief delay. Importantly, spatial location varied in a pseudorandom trial-by-trial basis, thus requiring mice only to maintain information relevant to the present trial. Using both pathway-specific optogenetic inhibition experiments and directionality analyses of multi-site recordings, we found that different task phases (sample, delay and choice) exhibited distinct thalamo-prefrontal dependencies. While initial spatial sampling required no functional interactions between MD and $\mathrm{MPFC}$, spatial choice required a directional interaction from $\mathrm{MPFC}$ to MD. In comparison, the delay phase required reciprocal interactions across the two structures with inputs from MD to mPFC exerting a stronger influence on behavior. Strikingly, and despite this clear behavioral dependence, $\mathrm{mPFC}$ neurons showed no evidence for maintaining a spatial memory across the delay, although mPFC spatial coding was readily detected during both the sample and choice phases. However, a subset of mPFC neurons demonstrated elevated delay phase spiking that indicated correct performance and was highly dependent on functional MD inputs. In a direct comparison of spatially-tuned and delay-elevated mPFC neurons we observed a double dissociation in their activity dependence on ventral hippocampal (vHPC) and MD inputs. This effect was moreover specific to the task phase in which each population was feature-selective in the task, suggesting a circuit-specific role for MD inputs in sustaining prefrontal activity across working memory delays. Consistent with this idea we 
found that temporally-restricted enhancement of MD excitability during the delay is sufficient to improve working memory performance.

Combined, these findings demonstrate a functional dissociation of prefrontal substrates for spatial encoding, maintenance and retrieval of working memory. We conclude that while vHPC inputs to the mPFC support spatial encoding as previously shown ${ }^{18}$, MD inputs to the mPFC support the maintenance of working memory by stabilizing task-relevant prefrontal activity during the delay period, and that top-down signals from the mPFC back to the MD guide successful memory retrieval and/or action execution.

\section{RESULTS}

\section{Activity within topographical MD-mPFC connections is required for spatial working memory}

While the anatomy of MD-prefrontal circuitry is well described in primates ${ }^{19}$ and rats ${ }^{20,21}$, there have been comparatively fewer anatomical studies in the mouse. We therefore first sought to extend this literature by closely examining thalamo-prefrontal connectivity in the mouse. Using viral-mediated synaptophysin-GFP expression to visualize MD terminals, we observed that mouse MD makes extensive synaptic connections with multiple prefrontal areas, with particularly dense terminations in the $\mathrm{mPFC}$ and orbitofrontal cortex (OFC) (Fig. $1 \mathrm{a}, \mathrm{b})$.

To identify the MD substructures that are anatomically associated with these PFC regions in the mouse, we delivered the dual antereograde/retrograde tracers fluoro-emerald and fluororuby to either the OFC or mPFC (Supplementary Fig. 1a,b). We found minimal overlap in both the terminal fields of PFC projections to the MD and MD neurons with projections back to the medial and orbital walls of the PFC (Supplementary Fig. 1c). That is, while $\mathrm{mPFC}$ neurons primarily projected to and received input from the lateral and medial MD, OFC neurons predominately projected to and received input from the central MD (Supplementary Fig. 1d). Although our injections did not distinguish between dorsal and ventral aspects of $\mathrm{mPFC}$, we generally observed denser connectivity with lateral MD when dorsal mPFC was densely labeled, and denser connectivity with medial MD when ventral mPFC was densely labeled. As recently reported in the mosue ${ }^{22}$, this reflects the presence of two distinct and topographically organized MD-mPFC circuits. This topographic and reciprocal MD-PFC organization is consistent with anatomical findings in primates ${ }^{19}$ and rats $^{20,21}$.

Previous findings suggested that $\mathrm{mPFC}$ is the prefrontal region most relevant to spatial working memory on a delayed non-match to sample (DNMS) "win-shift" T-maze in which mice have to choose a spatial location that differs from the one they randomly sample prior to a delay period ${ }^{23,24}$ (Fig. 1c). We previously demonstrated that this task also relies on MD activity ${ }^{10}$. We therefore hypothesized that activity in MD-to-mPFC projections, but not MDto-OFC projections, would be required for task performance. To test this hypothesis we optogenetically inhibited MD terminals alternately in either the $\mathrm{mPFC}$ or OFC within the same animals (Fig. 1d). Both regions are sufficiently far apart such that light does not spread from one region to another, an observation we independently confirm by modeling the 
propagation of light in the mPFC according to our specific optogenetic parameters (Supplementary Fig. 2a). Terminal inhibition was achieved by delivering $532 \mathrm{~nm}$ light (10mW) via flat-tipped optical fibers (200um, $0.22 \mathrm{NA}$ ) to MD terminals expressing the membrane trafficking-enhanced variant of the proton pump archaerhodopsin (eArch3.0; referred to as eArch hereafter), which we have previously shown to be effective for inhibiting ventral hippocampal (vHPC) inputs to the $\mathrm{mPFC}^{18,25}$. eYFP was used to control for potential effects of light alone ${ }^{26}$. We found a robust impairment of task performance when MD terminals were inhibited within the MPFC for the duration of a trial (Fig. 1e). No effect was observed when MD terminals within OFC were inhibited in an identical manner (Fig. 1e). Interestingly, this effect was related to delay phase length, with terminal inhibition only producing deficits under a more demanding long delay (60s). We previously observed a similar delay-dependent deficit in spatial working memory when inhibiting MD cell bodies ${ }^{10}$. The present results indicate that this finding is due to the inhibition of MD neurons projecting to the $\mathrm{mPFC}$ but not those projecting to the OFC.

Given the dense reciprocal connectivity between the mPFC and the MD (Supplementary Fig. 1d), we next asked whether $\mathrm{mPFC}$ projections to MD were also necessary for working memory performance. We therefore virally expressed eArch or eYFP in mPFC, and implanted optical fibers over mPFC terminals in the MD. Inhibiting the mPFC-to-MD projection diminished task performance in a manner that depended on delay phase length, similar to inhibition of the reciprocal MD-to-mPFC projection (Fig. 1f,g). These results demonstrate involvement of reciprocal MD-mPFC circuits in spatial working memory, and raise the possibility that activity in these circuits could work in concert to support task performance.

\section{Different task phases require distinct functional interactions between MPFC and MD}

To understand the precise manner by which $\mathrm{mPFC}$ and MD connections engage in working memory, we first performed temporally-limited optogenetic inhibition of MD terminals in mPFC (MD-to-mPFC) and mPFC terminals in MD (mPFC-to-MD) during specific phases of the task. In these experiments, we restricted the delay phase length to the 60 s condition, and terminal inhibition was limited alternately to the sample, delay, or choice phase of the task (Fig. 2a). Inhibiting MD-to-mPFC during the sample or choice phases did not significantly impact performance (Fig. 2b). In contrast, MD-to-mPFC inhibition during the delay phase substantially diminished performance, an effect not seen with optical illumination in the absence of eArch (Fig. 2b). This effect was also unrelated to the disparity between task phase lengths, as limiting delay inhibition to $17 \mathrm{~s}$ (equivalent to the average sample phase duration) was also sufficient to impair behavioral performance (Supplementary Fig. 3). Our sample sizes (phase-specific inhibition: $\mathrm{eArch}=17$; $\mathrm{eYFP}=11 ; 17$ s inhibition: $\mathrm{eArch}=16$; eYFP=12) were a priori sufficiently powered to detect significant interactions based on our previously observed effect size when inhibiting vHPC inputs to the mPFC during the sample phase $^{18}$. However, given non-significant tendencies for a decrease in performance when inhibiting MD inputs to the $\mathrm{mPFC}$ (phase-specific inhibition: $\mathrm{p}=0.59, \beta=0.91 ; 17 \mathrm{~s}$ inhibition: $\mathrm{p}=0.21, \beta=0.73$ ), we cannot exclude the possibility for a Type II error if the true effect size is smaller than predicted. Irrespective, these results strongly indicate that activity 
in the MD-to-mPFC pathway is particularly required during the delay phase, potentially supporting the maintenance of working memory.

To investigate whether mPFC-to-MD engagement in the task was symmetrical to that of the MD-to-mPFC pathway, we performed temporally-limited inhibition of this pathway during prescribed phases of the task. We found that, while sample phase inhibition had no effect on behavior, delay phase inhibition led to diminished task performance that approached statistical significance ( $p=0.073, \beta=0.53$; Fig. $2 c$ ). Strikingly, choice phase inhibition robustly impaired task performance (Fig. 2c, right), despite the brief duration of inhibition received (Supplementary Fig. 3a). These asymmetric effects suggest that MD-mPFC pathways are differentially engaged in the task. Specifically, while the MD-to-mPFC pathway is required during the delay phase, possibly supporting working memory maintenance, mPFC-to-MD pathways may also be required, but to a lesser degree. In contrast, mPFC-to-MD dependence during spatial choice indicates that the MD may function as an output station for mPFC to exert its impact on either working memory retrieval, action execution, or both. As our manipulations impacted the entire MD and both ventral- and dorsal-mPFC (Supplementary Fig. 2), which respectively share reciprocal connections with medial and lateral $\mathrm{MD}^{22}$, it is possible these asymmetric effects may be attributable to topographically discrete MD-mPFC circuits.

The asymmetric effects on working memory performance obtained by MD-to-mPFC inhibition compared to mPFC-to-MD suggested that functional interactions between these circuits are directional, and may vary in a task phase-dependent manner. To directly test this predication, we analyzed data from simultaneous electrophysiological recordings of mPFC single-units and MD local field potentials (LFPs) during the task (Fig 3a). We used a lag analysis of mPFC units that were significantly phase-locked to filtered MD beta oscillations $(13-30 \mathrm{~Hz})$ in order to estimate the net direction of information flow between the two structures (Fig. 3b). During the sample phase, when inhibition of either circuit had no effect on behavior, there was no net directionality between the MD and mPFC (Fig. 3c $\mathrm{c}_{\mathrm{i}-\mathrm{ii}}$ ). During the delay phase, however, MD activity led mPFC activity. This directionality suggests a predominance of MD-to-mPFC influence during this phase, consistent with the behavioral impairment seen with MD-to-mPFC terminal inhibition (Fig. $3 \mathrm{~d}_{\mathrm{i}-\mathrm{ii}}$ ). Finally, during the choice phase, $\mathrm{mPFC}$ activity led the MD, a result also in line with the behavioral impact of inhibiting this pathway (Fig. $3 \mathrm{e}_{\mathrm{i}-\mathrm{ii}}$ ). Importantly, this pattern of net information flow was also observable when performing a lag analysis on cross-correlations of the instantaneous amplitudes of filtered MD and mPFC LFPs ${ }^{27}$ (Supplementary Fig. 4a). While LFP-LFP cross-correlations indicated no net direction flow between MD and mPFC during the sample phase, MD LFPs robustly led those in the mPFC during the delay phase, and mPFC LFPs predominately led those in MD during the choice phase (Supplementary Fig. 4b-d). Further strengthening the links between these observations and our behavioral results, we found that MD-to-mPFC terminal inhibition during the sample phase had no effect on the net directionality of phase-locking between structures (Fig. 3f), while delay phase terminal inhibition diminished MD leading activity during the delay (Fig. 3g), although not during the subsequent spatial choice (Fig. 3h). Together, these findings support the conclusion that during the delay phase, mPFC is dependent on functional MD input for the maintenance of 
working memory, while during the subsequent choice phase, $\mathrm{MPFC}$ outputs to the MD guide the retrieval and/or action execution of successfully maintained working memory plans.

\section{mPFC neurons show elevated spiking during the delay but no spatial tuning}

Having obtained recordings from multiple mPFC single-units during the task, we interrogated their spiking across task phases to determine which task variables they encoded and when. Consistent with our prior results ${ }^{18}$ and those of others ${ }^{28}$, we found that many mPFC neurons were spatially-tuned towards one maze location over another during both the sample and choice phases (Fig. 4). This was clearly observed in both single-unit examples (Fig. 4b) and across the entire population of mPFC units (Fig. $4 \mathrm{c}_{\mathrm{i}-\mathrm{iii}}$ ), with spatial tuning in individual neurons largely overlapping between sample and choice phases (Fig $4 \mathrm{c}_{\mathrm{iii}}$, inset). In contrast, we found no evidence for spatial tuning during the delay phase, whether analyzed by which arm was visited during the preceding sample phase (Fig. $4 \mathrm{c}_{\mathrm{ii}}$ ) or by which arm was chosen during the subsequent choice phase (Fig $4 c_{i i}$, inset). Moreover, spatial tuning was not altered by concurrent MD terminal inhibition during the sample and delay phases (Fig. $4 \mathrm{~d}_{\mathrm{i}-\mathrm{ii}}$ ), nor was it affected during the choice phase following inhibition during the preceding delay phase (Fig. $\left.4 \mathrm{~d}_{\mathrm{iii}}\right)$. The same was observed when restricting analyses to only neurons that were significantly spatially-tuned (Supplementary Fig. 5). Importantly, these results were not due to a failure of terminal inhibition, as eArch activation produced significant modulations in $\mathrm{mPFC}$ firing rates compared to light illumination without eArch (Supplementary Fig. 6). These findings confirm previous observations that in rodents, $\mathrm{mPFC}$ neurons do not represent goal arm locations in sustained firing during the delay phase of T-maze tasks ${ }^{18,28}$, and further demonstrate that spatial representations of arm location in mPFC neurons are not dependent on MD-to-mPFC activity.

We reasoned that while mPFC neurons do not explicitly encode spatial location during the delay phase, they may represent other variables critical for task performance. Consistent with this, we found a subset of mPFC neurons (266/891) that exhibited significant elevation in spiking during the delay relative to the inter trial interval (ITI), where behavioral conditions were equivalent yet mice were not required to maintain a working memory trace. Elevation in spiking for each neuron was not sustained; rather, each neuron exhibited a preferred temporal offset during the delay phase (Fig. 5a). As a population, this activity pattern tiled the entire delay phase, and semi-automated clustering of the data based on temporal correlations in firing revealed a Poisson-like distribution pattern characterized by a gradual decay and broadening of clustered sub-population peaks as the delay period progressed $\left(\mathrm{Fig} 5 \mathrm{~b}_{\mathrm{i}}\right)$. This pattern was not observed in a largely mutually exclusive group of mPFC neurons identified as having significantly suppressed delay activity (260/891), where the vast majority of peak suppression occurred within the first five seconds of the delay (201/260) (Supplementary Fig. 7). Moreover, shuffled versions of the entire data set both significantly reduced the number of neurons exhibiting delay-elevated activity and abolished the unique temporal structure of delay-elevated clusters (Fig $5 b_{i i-i i i}$ ). These findings were replicated in an independent data set of mPFC single units (Fig $5 \mathrm{c}-\mathrm{d}$ ), together indicating that delay-elevated neurons are not artifacts created by spiking on a small number of trials and that the observed firing pattern of clustered data does not emerge by chance. 
Temporally sparse and sequential activation of neural ensembles have previously been reported in rodents performing tasks assessing both working memory and interval timing ${ }^{29-33}$. To assess whether delay-elevated mPFC neurons represented information related to interval timing, we recorded single-units in the $\mathrm{mPFC}$ in mice performing the Tmaze at two distinct delays $-60 \mathrm{~s}$ and $20 \mathrm{~s}$. In contrast to previous findings that time coding neural populations scale their activity according to interval durations ${ }^{32}$, we found that only delay-elevated neurons with peaks in the first 20s of the 60s delay retained their temporal preference during the shorter 20s delay (Supplementary Fig. 8). We therefore hypothesized that delay-elevated $\mathrm{mPFC}$ activity may reflect the maintenance of a working memory-related representation across the delay phase. If true, we reasoned that delay-elevated activity should be attenuated on incorrect trials. Indeed, we observed diminished spiking in delay-elevated neurons in normalized firing rates across individual neurons (Fig. 6a $\mathrm{a}_{\mathbf{i}}$ ), across clustered subpopulations (Fig. $6 \mathrm{a}_{\mathrm{ii}}$ ), in raw data examples (Fig. $6 \mathrm{a}_{\mathrm{iii}}$ ), and in the ratio of incorrect/correct firing in neurons grouped by distinct temporal offsets $\left(\mathrm{Fig} 6 \mathrm{~d}_{\mathrm{i}}\right)$.

Given that MD input during the delay phase was necessary for task performance, we next asked whether they were also important for delay activity in the mPFC. Interestingly, MD terminal inhibition throughout the delay had temporally-specific effects on mPFC delayfiring. While delay-elevated firing in clusters with early temporal offsets were largely left intact, firing rate in middle and late clusters were substantially diminished (Fig $6 b_{\text {i-iii }}$; Fig. $6 \mathrm{~d}_{\mathrm{ii}}$ ). These results are not explained by an effect of light alone, as they were not observed in eYFP animals (Supplementary Fig. 9). Moreover, these findings are also not due to a nonspecific effect of removing excitatory drive to $\mathrm{MPFC}$ neurons, as terminal inhibition of vHPC inputs to the mPFC during the delay had no impact on delay-elevated firing (Fig $6 c_{i-i i i} ;$ Fig $\left.6 d_{i i i}\right)$. Indeed, the MD-dependence of delay-elevated mPFC activity is strikingly input- and task-phase specific. While activity in delay-elevated neurons was suppressed by MD terminal inhibition during the delay phase (Fig. $7 \mathrm{a}_{\mathrm{i}-\mathrm{ii}}$, right), activity in this same population was unaffected by MD terminal inhibition during the sample phase (Fig. $7 \mathrm{a}_{\mathrm{ii}}$, left). Moreover, these neurons were also not impacted by vHPC terminal inhibition in either sample or delay phases (Fig. $7 b_{i-i i}$ ). These results suggest that MD inputs are specifically critical for sustaining delay-elevated $\mathrm{mPFC}$ representations across the delay phase. Strengthening this notion, the largely non-overlapping group of spatially-tuned $\mathrm{mPFC}$ neurons (Supplementary Fig. 7b) exhibited activity-dependence on vHPC inputs during the sample phase (Fig. $7 b_{\text {iii }}$ ), but were independent of MD inputs in both sample and delay phases (Fig. $7 \mathrm{a}_{\mathrm{iii}}$ ). Together, these results reveal a double dissociation of the MDdependence and vHPC-dependence of delay-elevated and spatially-tuned mPFC neurons, respectively. Strikingly, the two populations only depend on their respective inputs when modulated by the task phase in which they are feature selective in the task.

If delay-elevated $\mathrm{mPFC}$ neurons represent task-relevant information that is exclusively dependent on MD inputs for their maintenance across the delay phase, we reasoned that facilitating MD activity should improve behavioral performance in a task-phase specific manner. To test this idea we utilized a stabilized step function opsin (SSFO), which is capable of broadly enhancing neural excitability in a temporally-restricted manner without explicitly controlling spike timing ${ }^{34}$. As such, we virally delivered SSFO (AAV2-CamKIIaSSFO-mCherry) to the MD, bilaterally implanted fiberoptics dorsal to it, and activated 
SSFO exclusively during either the sample or delay phases in mice performing the DNMS Tmaze. Strikingly, we found that enhancing MD excitability during the delay, but not sample, phase of the task improved behavioral performance (Fig. 7c). This finding is consistent with a specific role for MD inputs in sustaining delay-elevated $\mathrm{mPFC}$ activity without impacting mPFC spatial encoding during the sample phase and provides further evidence that MDdependent delay-elevated mPFC activity supports the maintenance of working memory representations critical for task performance.

\section{DISCUSSION}

Here we demonstrate that reciprocal MD-mPFC activity is required for spatial working memory in mice (Fig. 1). By dissecting the role of each reciprocal projection during discrete task phases of the DNMS T-maze we further reveal that while MD inputs to the mPFC support the maintenance of working memory, mPFC inputs to the MD support the retrieval of memory for action execution (Fig. 2). We corroborate this model using two distinct directionality analyses of simultaneous MPFC and MD activity, which both revealed a dynamic shift from MD leading activity during the delay phase towards mPFC leading activity during the choice phase (Fig. 3 and Supplementary Fig. 4). Finally, we uncover a population of $\mathrm{mPFC}$ neurons whose activity is temporally sparse within individual neurons, and sequential across the population (Fig. 5). This activity pattern indicates correct performance and depends on MD inputs, but not vHPC inputs, for sustained maintenance across the delay (Fig. 6). We further reveal a double dissociation of the MD-dependence and vHPC-dependence of delay-elevated and spatially-tuned mPFC neurons, a finding that is also specific to the task phase in which each population is feature selective (Fig. 7). These findings are consistent with a role for MD inputs in sustaining $\mathrm{mPFC}$ working memory representations across a delay, a notion supported by improvements in working memory performance when enhancing MD activity during the delay, but not sample, phase of our working memory task (Fig. 7).

What is the nature of the mPFC representation supported by MD input during the delay? The absence of spatial-tuning across our mPFC population during the delay (Fig. 4), the largely exclusive nature of spatially-tuned and delay-elevated mPFC populations (Supplementary Fig. 7b) and the vHPC-independence of delay-elevated activity (Fig. $7 b_{\text {i-ii }}$ ) strongly argues against the presence of an explicit spatial representation. This finding is surprising in the context of well-documented observations of spatial-tuning in PFC delay period activity in primates ${ }^{35-37}$. This is typically observed in delayed response tasks requiring the cacheing of one spatial location from multiple potential targets. Interestingly, in a two-choice version of this task analogous to ours, seminal work demonstrating sustained delay period activity in primate PFC also reported that spatial preference in delay-tuned neurons was either absent ${ }^{38}$ or minimal ${ }^{39,40}$ (6-13\% of sustained delay units). mPFC delay activity does also not appear to encode timing of the delay interval, as delay-elevated mPFC neurons do not scale their activity according to distinct delay durations (Supplementary Fig. 8), a feature observed in neural ensembles explicitly linked to interval timing ${ }^{32}$. Delay-elevated activity may instead reflect a general attentive or task-engaged state. Although our findings do not exclude this possibility, we did not observe overt behavioral differences between correct and incorrect trials, nor was the latency for mice to make a spatial choice altered by MD terminal 
inhibition (data not shown). A final possibility is that explicit spatial representations are unnecessary for two-choice spatial working memory tasks, and that an abstract task rule, such as 'go to opposite location', is sufficient to guide behavioral performance. A direct test of this hypothesis within the context of our task however, would require task rule, not only spatial location, to vary on a trial-by-trial basis.

Nevertheless, task rule encoding in PFC neurons has been frequently reported in both rodents ${ }^{41,42}$ and primates ${ }^{43,44}$. Moreover, findings from a recent working memory guided, top-down attention task, in which task rules are varied on a trial-by-trial basis, explicitly demonstrate task rule encoding in mouse mPFC neurons during the delay period ${ }^{45}$. Similar to the delay-elevated mPFC activity in our task, this study observes temporally sparse and sequential mPFC spiking that codes for one of two task rules during the delay period. Importantly, further strengthening our inference, delay-elevated activity in this task requires MD activity for its sustained maintenance across the delay. Our results make clear this effect is due to activity in MD-to-mPFC projections, and is not due to alterations in general excitability.

The parallels between our findings and Schmitt et al. ${ }^{45}$ are quite striking when considering the difference in temporal scale between tasks. The fact that our population of mPFC neurons was capable of spanning a 60s delay may indicate a fundamental scalability of mPFC encoding and maintenance of rule representation across time scales. In this light, our findings extend studies that have identified sequential activation of cortical neurons in tasks that require working memory ${ }^{29-31}$, and support the idea that sequences of activation may be a common circuit function in memory-guided decision tasks. It is also interesting to note that we observed a gradual degradation in the quality of elevated spiking across time (Fig. 5a,b). This is evocative of a decay in prefrontal representation, and may explain why our MDmPFC manipulations did not impact behavior at shorter delays (Fig. 1e, left and Fig. 1g, left), where delay representations were potentially robust to activity disruptions in our task. Further consistent with this interpretation is the fact that mPFC delay activity with early peak times were unaffected by MD terminal inhibition (Fig 6b, $\mathrm{d}_{\mathrm{ii}}$ and Fig $7 \mathrm{a}_{\mathrm{ii}}$ ). Local PFC circuitry may therefore be sufficient to maintain representations at short time-scales, but require amplification for sustained maintenance either as memory decays across time, or in more cognitively demanding tasks.

How does the mPFC-to-MD pathway support working memory performance? Our terminal inhibition experiments and directionality analyses both indicate a critical role for this pathway during the choice phase of our task, when mice presumably require the retrieval of maintained information and its translation to motor action (Fig. 2c; Fig. 3e $\mathrm{e}_{\mathrm{i}-\mathrm{ii}}$;

Supplementary Fig. 4). Although this finding may suggest a surprising functional dissociation with the reciprocal MD-to-mPFC pathway, this interpretation warrants significant caution. Our pathway-specific experiments do not distinguish between dorsal and ventral mPFC (Supplementary Fig. 2), which reciprocally connect with lateral and medial MD in a largely segregated manner ${ }^{21,22}$. It is therefore possible that the distinct phasespecific behavioral impairments we observe may be attributable to activity in discrete MDmPFC circuits, a possibility that will be important to resolve in future research.

Nevertheless, this observation does suggest that the lateral and/or medial MD may serve as 
an intermediate output pathway for mPFC to recruit downstream motor planning circuits. Indeed, the mPFC has been shown to exert strong functional control over primary motor corte ${ }^{46}$, and higher order thalamic nuclei like the MD are posited to play a role in information transfer between cortical areas ${ }^{6,47}$. While anatomical connectivity exists between MD and primary and secondary motor cortex ${ }^{48,49}$, the behavioral function of these circuits remain entirely unexplored. An alterative possibility is that mPFC-to-MD circuits may be important for cortical or subcortical pathways involved in memory retrieval. Future efforts to expand functional circuit dissection of MD-PFC pathways and their associated structures with increasing precision could provide answers to these important questions.

In the context of our previous studies inhibiting vHPC-to-mPFC inputs ${ }^{18}$, our results provide a striking example of differential long-range circuit engagement in the DNMS T-maze. Here we observed limited impact of MD terminal inhibition restricted to the sample phase of the task and a robust behavioral impairment with inhibition restricted to the delay phase (Fig. 2b and Supplementary Fig. 3). In contrast, vHPC terminal inhibition specifically during the sample, but not delay phase robustly impairs behavioral performance ${ }^{18}$. Extending this dissociation further, we reveal here a double dissociation in the MD-dependence and vHPCdependence of delay-elevated and spatially-tuned mPFC neurons (Fig. 7a,b). This finding demonstrates a functional dissociation of prefrontal substrates for working memory encoding and maintenance in the DNMS T-maze task. Our results suggest this dissociation is largely due to the segregation of spatially-tuned and delay-elevated mPFC neurons into largely non-overlapping populations (Supplementary Fig. 7b).

Our findings should have translational relevance, particularly to schizophrenia. Patients with schizophrenia exhibit prefrontal-associated cognitive deficits in domains such as executive function and working memory and neuroimaging studies increasingly report diminished thalamo-prefrontal connectivity ${ }^{15-17}$. Our data provide clear evidence that these circuit abnormalities are likely to be causally involved in producing working memory deficits. Continued investigation of thalamo-prefrontal interactions in different behavioral conditions in patients and in animal models will be critical for advancing clinical efforts for improved diagnoses and more targeted therapeutic approaches ${ }^{50}$.

\section{ONLINE METHODS}

\section{Animals}

All experiments were carried out on male C57/B16 male mice purchased from Jackson Laboratory. Mice were aged 7-8 weeks at the start of experiments and housed under a 12 hour, light-dark cycle in a temperature controlled environment with food and water available ad libitum. For optogenetic experiments, mice were group housed with littermates (5 mice/ cage). Mice with implanted microdrives were individually housed. During behavioral training and testing, mice were food restricted and maintained at $85 \%$ of their initial weight. All procedures were done in accordance with guidelines derived from and approved by the Institutional Animal Care and Use Committees at Columbia University and the New York State Psychiatric Institute. 


\section{Surgical Procedures}

Mice were first anesthetized with isoflurane and head-fixed in a stereotactic apparatus (Kopf). In anatomical tracing experiments, an AAV1 expressing synaptophysin-eGFP under the chicken beta actin (CBA) promoter was injected unilaterally into the MD at a volume of $0.2 \mathrm{ul}(0.1 \mathrm{ul} / \mathrm{min})$. Four mice were used and results were identical across all. Viral production was carried out at Columbia University and shared care of the laboratory of Thomas Jessell. Dextran-amine coupled fluorophore tracers (fluoro-Ruby and fluoro-Emerald) were obtained commercially (ThermoFisher Scientific) and bilaterally injected into the mPFC or OFC at a volume of $0.4 \mathrm{ul}(0.1 \mathrm{ul} / \mathrm{min})$. Four mice were used and results were identical across all. In optogenetic inhibition experiments, mice were bilaterally injected in the MD or mPFC with an AAV5 expressing either eYFP alone, or an eArch3.0-eYFP fusion construct under the hSynapsin promoter at a volume of 0.25 or $0.35 \mathrm{ul}$, respectively $(0.1 \mathrm{ul} / \mathrm{min})$. vHPC was targeted with four injection sites per hemisphere at a volume of $0.2 \mathrm{ul}$ each $(0.1 \mathrm{ul} / \mathrm{min})$. In SSFO experiments, AAV2-CaMKIIa-hChR2(C128S/D156A)-mCherry was delivered bilaterally to the $\mathrm{MD}$ at a volume of $0.4 \mathrm{ul}(0.1 \mathrm{ul} / \mathrm{min})$. Opsin-expressing virus was obtained commercially from the University of North Carolina Viral Vector Core. Viral and tracer coordinates were as follows: MD coordinates (-1.2 AP, $-3.2 \mathrm{DV}$ skull, $+/-0.35 \mathrm{ML})$; mPFC coordinates (1.75 AP, $-1.8 \mathrm{DV}$ brain, +/-0.4 ML), vHPC coordinates (-3.0 AP, +/-3.25, -4.0 and $-1.75 \mathrm{DV} ;-3.0 \mathrm{AP},+/-2.5,-3.0 \mathrm{DV} ;-3.0 \mathrm{AP},+/-3.4,-3.0 \mathrm{DV})$.

In optogenetic experiments, in the same procedure mice were also bilaterally implanted with flat tipped, ferrule-coupled optical fibers ( $0.22 \mathrm{NA}, 200$ um diameter) immediately dorsal to the targeted structure (OFC, mPFC, or MD), which were fixed to the skull with dental cement. Coordinates were as follows: OFC ( $+2.65 \mathrm{AP},-2.25 \mathrm{DV}$ skull, $+/-1.85 \mathrm{ML}), \mathrm{mPFC}$ (+1.75 AP, -1.2 DV brain, +/- 0.4 ML), and MD (-1.2 AP, -2.75 DV skull, +/- 0.25 ML). Coordinates are in mm relative to bregma (AP, ML) and skull or brain surface (DV) where specified.

For in vivo neurophysiology experiments, mice were implanted with a moveable microdrive consisting of a 32-channel electronic interface board (NeuroNexus), bilateral ferrule-coupled optical fibers (center-to-center distance: 700-800um), and a single stereotrode bundle.

Stereotrodes for recording spikes were made from 13-uM tungsten fine wire (California Fine Wire, Grover Beach, CA) and were coupled to the optical fiber such that stereotrode tips were positioned $300-500 \mathrm{uM}$ ventral to the fiber tip. The fiber-coupled stereotrode bundle was then unilaterally targeted to the left mPFC. An additional 50uM tungsten wire for recording LFPs was implanted in the left MD and fixed to the skull with dental cement. For LFP signal processing, skull screws placed over the cerebellum and olfactory bulb served as ground and reference, respectively, while spikes were referenced to a local mPFC stereotrode wire. The microdrive was lowered in $80 \mathrm{uM}$ steps between recording sessions until reaching a depth of $-2 \mathrm{~mm}$.

\section{Behavior}

Following $\sim 5$ weeks of viral expression, mice were gradually food restricted to $85 \%$ of their body weight. Mice were then given 2 days of habituation to the T-maze, which consisted of $10-20 \mathrm{~min}$ of free exploration and foraging for food rewards while tethered to optical fibers 
and/or the recording tether. On the subsequent 2 days mice underwent behavioral shaping, which consisted of 10 runs from the start box to a baited goal arm and back to the start box. Runs were forced choice in alternating directions and mice were habituated to laser illumination on half of the runs in a random interleaved fashion. Mice then commenced training on the DNMS T-maze for 6 consecutive days without laser illumination. Unlike the delayed alternation T-maze task, sample arm runs were pseudo-randomly selected on a trialby-trial basis. Within this window, all mice reached a criterion level of performance defined as 3 consecutive days above $70 \%$ correct. During the subsequent testing phase, in all experiments laser illumination was delivered in a randomly interleaved fashion and with even distribution across trial types and animals. The experimenter was blind to the viral condition of mice during behavioral testing. For whole trial light experiments, testing at $10 \mathrm{~s}$ and 60s delays occurred on separate days. For physiology experiments comparing mPFC activity at 20s and 60s delays, testing was performed within the same session. For behavior only and neurophysiology experiments reward consisted of either dustless pellets (Bio-Serv) or sweetened condensed milk ( $\sim \mathrm{ul}, 3: 1$ dilution), respectively. The inter-trial interval for all experiments was fixed at 40s. All behavior was conducted during the light cycle.

\section{Optogenetic Parameters}

Pathway-specific optogenetic inhibition experiments were carried out using $10 \mathrm{~mW}, 532 \mathrm{~nm}$ constant light, delivered via flat tipped 200 um diameter, 0.22 NA fiber optics. In SSFO experiments, a $50 \mathrm{~ms}$ blue light pulse $(473 \mathrm{~nm}, 4 \mathrm{~mW})$ was used for opsin activation and a $50 \mathrm{~ms}$ yellow light pulse $(593 \mathrm{~nm}, 4 \mathrm{~mW})$ was used for opsin deactivation. Light output from fiber optics was predicted using a Monte Carlo modeling approach as previously published ${ }^{26}$. Absorption and scattering coefficients for $532 \mathrm{~nm}$ light were interpolated from data measured in vivo ${ }^{51}$. The predicted fluence rate was calculated according to our $10 \mathrm{~mW}$ output from $200 \mathrm{um}, 0.22 \mathrm{NA}$ fibers into a large cubic volume $\left(6 \mathrm{~mm}^{3}\right)$ of gray matter. The volume with a fluence rate above $7.5 \mathrm{~mW} / \mathrm{mm}^{2}$, the approximate EPD50 of eArch3.0 ${ }^{52}$, was calculated and plotted to scale on MD and $\mathrm{mPFC}$ brain slices from a mouse stereotactic reference atlas ${ }^{53}$ to predict the effectively inhibited volume. Based on the model and our viral expression pattern and fiber optic targeting, in all mice $\mathrm{mPFC}$ projections to medial, central and lateral MD, and MD projections to dorsal and ventral MPFC were in part effectively inhibited. In our MD-to-mPFC task phase-specific experiments, our viral spread typically included expression in the paraventricular nucleus of the thalamus (PVT).

However, viral spread in 5 of 33 mice was confirmed to spare the PVT. Our reported MD-tomPFC delay-phase inhibition effect was clearly observable in 4 of the 5 mice (Light OFF: $75 \%+/-2.1$; ON Delay: $67 \%+/-2.0$ ).

\section{Data Acquisition}

Recordings were amplified, band-pass filtered (1-1000 Hz LFPs; 600-6000 Hz spikes) and digitized using a Digital Lynx system (Neuralynx). LFPs were collected at $2 \mathrm{kHz}$, while spikes were detected by online thresholding, collected at $32 \mathrm{kHz}$, and sorted off-line. Single units were automatically clustered using Klustakwik (Ken Harris) based on spike sorting of the first two principal components, peak voltage and energy from each stereotrode channel. Clusters were then accepted, merged or removed based on isolation distance, visual 
inspection of feature segregation, inter-spike interval distribution, cross-correlation in spike timing for simultaneously recorded units, and stability across recording session.

\section{Single-Unit Analysis}

In MD-to-mPFC experiments we isolated a total of 891 and 686 single-units in 9 eArch and 9 eYFP animals, respectively. 538 eArch and 447 eYFP units were considered well-isolated, while the remainder of clusters shared modest contamination with multi-unit activity. To assess significantly light-modulated units, we considered only well-isolated clusters. We found that MD-to-mPFC inhibition significantly decreased (17\%, 92 single-units) and increased (15\%, 83 single-units) firing rates. This is consistent with monosynaptic inhibition and polysynaptic disinhibition of cortical projection neurons via fast-spiking interneurons, as has been described for prefrontal projecting MD neurons in the mouse ${ }^{54}$. Results were similar when all clusters were included (136/891 decrease, 134/891 increase in eArch; 52/686 decrease and 56/686 increase in eYFP). Significantly light-modulated mPFC singleunits were determined using bootstrapping. Specifically, light off and light on spike trains were randomly shuffled 30,000 times. If the observed light off/on firing rate difference was greater than $95 \%$ of the firing rate difference from shuffled data, single-units were deemed light-responsive. For all analyses of task-modulated single-unit activity, we included all 891 eArch and 686 eYFP units.

In vHPC-to-MD experiments we isolated a total of 800 single-units in 6 eArch-expressing mice. In this cohort of mice we carried out within session testing at 60 s delays with interleaved light off, light on sample, light on delay trials, followed by light off testing at $20 \mathrm{~s}$ delays. 657/800 units were stable, well-isolated, and retained cluster features across 60s and 20 s testing and were therefore included for analysis in Supplementary Fig. 9. All 800 units were used for analysis of task-modulated activity with and without vHPC-to-mPFC inhibition.

The preferred arm of single-units was determined from the mean firing rate $+/-500 \mathrm{~ms}$ around goal arrival on all left-visited versus right-visited trials in the sample or choice task phases. The observed preference during either sample or choice arm visits was then used for delay phase activity. Z-scored firing rates for arm preference were then calculated in $100 \mathrm{~ms}$ (sample and choice phases) or 1s (delay phase) bins based on the mean bin $\mathrm{x}$ bin firing rates across ITIs and the standard deviation between bins. Average firing rate $+/-500 \mathrm{~ms}$ of sample goal arrival on all left versus right trials was used to determine significance of spatial tuning across all mPFC neurons (Wilcoxon's rank-sum test, $\mathrm{p}<0.05$ ).

Delay-modulated activity was determined from z-scored firing rates calculated in 1s bins based on the mean bin $\mathrm{x}$ bin firing rate across ITIs and the standard deviation between bins. If single-units exhibited a $\mathrm{z}$-scored firing rate beyond $+/-2$ standard deviations for two consecutive bins or more, it was classified as a delay-elevated or delay-suppressed unit, respectively. The same criterion was used on shuffled versions of the entire MD-to-mPFC and vHPC-to-mPFC data sets. Shuffling was performed in a trial-by-trial manner that preserved the temporal structure of spikes. Specifically, each trial spike train was treated as a continuous circular vector, and a randomly selected time point in each was designated as time zero. This was performed 1000 times for each trial across all neurons. 
The pairwise distance in firing rate across time in delay-elevated neurons was used for clustering into six groups using the kmeans function in MATLAB. The percentage of variance explained as a function of the number of clusters was used to estimate optimal cluster number. Six clusters were sufficient to explain $\sim 50 \%$ of the variance and parceling the data into greater or fewer than six clusters neither improved visualization of groups nor altered the observed effects.

\section{Directionality Analyses}

Functional directionality based on MPFC spikes and MD LFP was performed by successively calculating the pairwise phase comparison (PPC) ${ }^{55}$ of mPFC spikes to MD LFP when shifting $\mathrm{mPFC}$ spikes in $10 \mathrm{~ms}$ steps $+/-100 \mathrm{~ms}$. MD LFP signal was first digitally band-pass filtered (13-30 Hz) using a zero-phase-delay filter (filter0, provided by K. Harris and G. Buzsaki) and the Hilbert transform of the bandpass-filtered signal was calculated to obtain oscillatory phase. The magnitude of phase-nonuniformity of spike times relative to the filtered LFP oscillation was then calculated at each temporal offset during each task phase (sample, delay, or choice). Only single-units that exhibited Bonferonni-corrected PPC values at peak lag (Rayleigh's circular test, $\mathrm{p}<0.05 / 21$ ) were used for analysis. In order to avoid spuriously high or low PPC values, only units that fired at least 100 spikes per condition were used. We chose to bandpass filter in the beta frequency range given our previous results showing task modulation of MD-mPFC beta synchrony ${ }^{9}$. While we did not observe effects of task phase on directionality when filtering at low $(40-70 \mathrm{~Hz})$ or high $(70$ $120 \mathrm{~Hz})$ gamma bands, filtering at theta frequency $(4-12 \mathrm{~Hz})$ produced results similar to those reported here. Prior to commencing analysis, we excluded 344 single-units due to improperly placed LFP electrodes or significant noise contamination in LFP signal during recording sessions.

Functional directionality based on MD and mPFC LFPs was performed as previously described ${ }^{27}$. Briefly, MD and mPFC LFP were bandoass-filtered as described above. The instantaneous amplitude for all points in the MD and LFP signal was calculated and the cross-correlation between amplitudes of the two signals was computed using the MATLAB function $x$ corr. This was done over lags ranging $+/-100 \mathrm{~ms}$ in $1 \mathrm{~ms}$ shifts. 90 recording sessions were included in the analysis.

\section{Statistics}

A two-way repeated measures ANOVA was used to assess significant interactions of light and virus in all behavioral experiments. Throughout, where significant interactions emerged, post-hoc two-tailed t-tests were performed for paired comparisons between light off and light on conditions, unless otherwise stated. When data was non-parametric we used Wilcoxon's signed-rank and rank-sum tests for paired and unpaired observations, respectively. Sample sizes for all experiments were based on previous work ${ }^{10,18}$ and were reported in all figure legends along with the $p$ values for all statistical comparisons. Where appropriate, Bonferroni-corrected $p$ values were used and indicated for multiple comparisons. For the comparison of proportions we used the two-sample KolmogorovSmirnov goodness-of-fit test. For linear regression fit of $60 \mathrm{~s} / 20$ s peak firing times we used 
the MATLAB function fitlm to perform a robust regression using the bisquare weighting function.

\section{Histology}

At the end of experimentation, mice were transcardially perfused with PBS followed by $4 \%$ PFA. For neurophysiology experiments, electrolytic lesions were induced at each recording site by passing current $(50 \mathrm{uA}, 20 \mathrm{~s})$ through electrodes prior to perfusion. Fixed tissue was then sectioned $(50 \mathrm{uM})$ using a vibratome, and mounted on slides with Vectashield mounting medium containing DAPI (Vector Labs). Direct fluorescence of eArch-eYFP or eYFP was then examined under an epifluorescent microscope (Zeiss) to assess extent of viral spread and axon terminal expression pattern. Locations of recording site lesions were confirmed with visualization under DAPI (Supplementary Fig. 10 and Supplementary Fig. 11). Two mice were excluded from MD LFP analyses due to failed electrode targeting.

\section{Data Availability}

The data that support the findings of this study are available from the corresponding author upon reasonable request.

\section{Code availability}

MATLAB code used for analysis of the data that support the findings of this study is available from the corresponding author upon request.

\section{Supplementary Material}

Refer to Web version on PubMed Central for supplementary material.

\section{Acknowledgments}

We would like to thank members of the Gordon and Kellendonk labs for technical assistance and discussions. We also thank M. Halassa for discussions and commentary on an initial draft of the manuscript. This work was supported by grants from the NIMH R01 MH096274 (J.A.G), F31 MH102041 (S.S.B) and F30 MH107204 (J.M.S.); by the Hope for Depression Research Foundation (J.A.G.); and by the Irma Hirschl Trust (C.K.).

\section{References}

1. Fuster, JM. Anatomy, Physiology, and Neuropsychology of the Frontal Lobe. 2. Raven; New York: 1989. The Prefrontal Cortex.

2. Minzenberg MJ, Laird AR, Thelen S, Carter CS, Glahn DC. Meta-analysis of 41 functional neuroimaging studies of executive function in schizophrenia. Arch Gen Psychiatry. 2009; 66:811822. DOI: 10.1001/archgenpsychiatry.2009.91 [PubMed: 19652121]

3. Weinberger DR, Berman KF. Prefrontal function in schizophrenia: confounds and controversies. Philos Trans R Soc Lond B Biol Sci. 1996; 351:1495-1503. DOI: 10.1098/rstb.1996.0135 [PubMed: 8941961]

4. Perlstein WM, Carter CS, Noll DC, Cohen JD. Relation of prefrontal cortex dysfunction to working memory and symptoms in schizophrenia. Am J Psychiatry. 2001; 158:1105-1113. DOI: 10.1176/ appi.ajp.158.7.1105 [PubMed: 11431233]

5. Mitchell AS. The mediodorsal thalamus as a higher order thalamic relay nucleus important for learning and decision-making. Neuroscience and biobehavioral reviews. 2015; 54:76-88. [PubMed: 25757689] 
6. Saalmann YB. Intralaminar and medial thalamic influence on cortical synchrony, information transmission and cognition. Front Syst Neurosci. 2014; 8:83. [PubMed: 24847225]

7. Baxter MG. Mediodorsal thalamus and cognition in non-human primates. Front Syst Neurosci. 2013; 7:38. [PubMed: 23964206]

8. Jones, EG. The Thalamus. 2. Cambridge University Press; New York: 2007.

9. Parnaudeau S, et al. Mediodorsal thalamus hypofunction impairs flexible goal-directed behavior. Biol Psychiatry. 2015; 77:445-453. DOI: 10.1016/j.biopsych.2014.03.020 [PubMed: 24813335]

10. Parnaudeau $\mathrm{S}$, et al. Inhibition of mediodorsal thalamus disrupts thalamofrontal connectivity and cognition. Neuron. 2013; 77:1151-1162. DOI: 10.1016/j.neuron.2013.01.038 [PubMed: 23522049]

11. Browning PG, Chakraborty S, Mitchell AS. Evidence for Mediodorsal Thalamus and Prefrontal Cortex Interactions during Cognition in Macaques. Cereb Cortex. 2015; 25:4519-4534. DOI: 10.1093/cercor/bhv093 [PubMed: 25979086]

12. Bailey KR, Mair RG. Lesions of specific and nonspecific thalamic nuclei affect prefrontal cortexdependent aspects of spatial working memory. Behav Neurosci. 2005; 119:410-419. DOI: 10.1037/0735-7044.119.2.410 [PubMed: 15839787]

13. Byne W, Hazlett EA, Buchsbaum MS, Kemether E. The thalamus and schizophrenia: current status of research. Acta Neuropathol. 2009; 117:347-368. DOI: 10.1007/s00401-008-0404-0 [PubMed: 18604544]

14. Andrews J, Wang L, Csernansky JG, Gado MH, Barch DM. Abnormalities of thalamic activation and cognition in schizophrenia. Am J Psychiatry. 2006; 163:463-469. DOI: 10.1176/appi.ajp. 163.3.463 [PubMed: 16513868]

15. Woodward ND, Karbasforoushan H, Heckers S. Thalamocortical dysconnectivity in schizophrenia. Am J Psychiatry. 2012; 169:1092-1099. DOI: 10.1176/appi.ajp.2012.12010056 [PubMed: 23032387]

16. Anticevic A, et al. Characterizing thalamo-cortical disturbances in schizophrenia and bipolar illness. Cereb Cortex. 2014; 24:3116-3130. DOI: 10.1093/cercor/bht165 [PubMed: 23825317]

17. Anticevic A, et al. Association of Thalamic Dysconnectivity and Conversion to Psychosis in Youth and Young Adults at Elevated Clinical Risk. JAMA Psychiatry. 2015; 72:882-891. [PubMed: 26267151]

18. Spellman T, et al. Hippocampal-prefrontal input supports spatial encoding in working memory. Nature. 2015; 522:309-314. DOI: 10.1038/nature14445 [PubMed: 26053122]

19. Ray JP, Price JL. The organization of projections from the mediodorsal nucleus of the thalamus to orbital and medial prefrontal cortex in macaque monkeys. J Comp Neurol. 1993; 337:1-31. DOI: 10.1002/cne.903370102 [PubMed: 7506270]

20. Groenewegen HJ. Organization of the afferent connections of the mediodorsal thalamic nucleus in the rat, related to the mediodorsal-prefrontal topography. Neuroscience. 1988; 24:379-431. [PubMed: 2452377]

21. Alcaraz F, Marchand AR, Courtand G, Coutureau E, Wolff M. Parallel inputs from the mediodorsal thalamus to the prefrontal cortex in the rat. Eur J Neurosci. 2016; 44:1972-1986. DOI: 10.1111/ ejn.13316 [PubMed: 27319754]

22. Matyas F, Lee J, Shin H, Acsady L. The fear circuit of the mouse forebrain: connections between the mediodorsal thalamus, frontal cortices and basolateral amygdala. European Journal of Neuroscience. 2014; 39:1810-1823. [PubMed: 24819022]

23. Kellendonk C, et al. Transient and selective overexpression of dopamine D2 receptors in the striatum causes persistent abnormalities in prefrontal cortex functioning. Neuron. 2006; 49:603615. DOI: 10.1016/j.neuron.2006.01.023 [PubMed: 16476668]

24. Yoon T, Okada J, Jung MW, Kim JJ. Prefrontal cortex and hippocampus subserve different components of working memory in rats. Learn Mem. 2008; 15:97-105. DOI: 10.1101/lm.850808 [PubMed: 18285468]

25. Padilla-Coreano N, et al. Direct Ventral Hippocampal-Prefrontal Input Is Required for AnxietyRelated Neural Activity and Behavior. Neuron. 2016; 89:857-866. [PubMed: 26853301] 
26. Stujenske JM, Spellman T, Gordon JA. Modeling the Spatiotemporal Dynamics of Light and Heat Propagation for In Vivo Optogenetics. Cell Rep. 2015; 12:525-534. DOI: 10.1016/j.celrep. 2015.06.036 [PubMed: 26166563]

27. Adhikari A, Sigurdsson T, Topiwala MA, Gordon JA. Cross-correlation of instantaneous amplitudes of field potential oscillations: a straightforward method to estimate the directionality and lag between brain areas. J Neurosci Methods. 2010; 191:191-200. [PubMed: 20600317]

28. Jung MW, Qin Y, McNaughton BL, Barnes CA. Firing characteristics of deep layer neurons in prefrontal cortex in rats performing spatial working memory tasks. Cereb Cortex. 1998; 8:437450. [PubMed: 9722087]

29. Fujisawa S, Amarasingham A, Harrison MT, Buzsaki G. Behavior-dependent short-term assembly dynamics in the medial prefrontal cortex. Nat Neurosci. 2008; 11:823-833. DOI: 10.1038/nn.2134 [PubMed: 18516033]

30. Harvey CD, Coen P, Tank DW. Choice-specific sequences in parietal cortex during a virtualnavigation decision task. Nature. 2012; 484:62-68. DOI: 10.1038/nature10918 [PubMed: 22419153]

31. Baeg EH, et al. Dynamics of population code for working memory in the prefrontal cortex. Neuron. 2003; 40:177-188. [PubMed: 14527442]

32. Mello GB, Soares S, Paton JJ. A scalable population code for time in the striatum. Curr Biol. 2015; 25:1113-1122. DOI: 10.1016/j.cub.2015.02.036 [PubMed: 25913405]

33. Akhlaghpour H, et al. Dissociated sequential activity and stimulus encoding in the dorsomedial striatum during spatial working memory. Elife. 2016:5.

34. Yizhar O, et al. Neocortical excitation/inhibition balance in information processing and social dysfunction. Nature. 2011; 477:171-178. DOI: 10.1038/nature10360 [PubMed: 21796121]

35. Goldman-Rakic PS. Cellular basis of working memory. Neuron. 1995; 14:477-485. [PubMed: 7695894]

36. Funahashi S. Space representation in the prefrontal cortex. Prog Neurobiol. 2013; 103:131-155. [PubMed: 22521602]

37. Rao SC, Rainer G, Miller EK. Integration of what and where in the primate prefrontal cortex. Science. 1997; 276:821-824. DOI: 10.1126/Science.276.5313.821 [PubMed: 9115211]

38. Fuster JM. Unit activity in prefrontal cortex during delayed-response performance: neuronal correlates of transient memory. J Neurophysiol. 1973; 36:61-78. [PubMed: 4196203]

39. Niki H. Differential activity of prefrontal units during right and left delayed response trials. Brain Res. 1974; 70:346-349. [PubMed: 4207718]

40. Niki H. Prefrontal unit activity during delayed alternation in the monkey. I. Relation to direction of response. Brain Res. 1974; 68:185-196. [PubMed: 4363757]

41. Rich EL, Shapiro M. Rat prefrontal cortical neurons selectively code strategy switches. J Neurosci. 2009; 29:7208-7219. DOI: 10.1523/JNEUROSCI.6068-08.2009 [PubMed: 19494143]

42. Durstewitz D, Vittoz NM, Floresco SB, Seamans JK. Abrupt transitions between prefrontal neural ensemble states accompany behavioral transitions during rule learning. Neuron. 2010; 66:43844866. [PubMed: 20471356]

43. Wallis JD, Anderson KC, Miller EK. Single neurons in prefrontal cortex encode abstract rules. Nature. 2001; 411:953-956. DOI: 10.1038/35082081 [PubMed: 11418860]

44. Cromer JA, Roy JE, Miller EK. Representation of multiple, independent categories in the primate prefrontal cortex. Neuron. 2010; 66:796-807. DOI: 10.1016/j.neuron.2010.05.005 [PubMed: 20547135]

45. Schmitt LI, et al. Sustaining cortical representations by a content-free thalamic amplifier. (In submission).

46. Narayanan NS, Laubach M. Top-down control of motor cortex ensembles by dorsomedial prefrontal cortex. Neuron. 2006; 52:921-931. DOI: 10.1016/j.neuron.2006.10.021 [PubMed: 17145511]

47. Sherman SM, Guillery RW. The role of the thalamus in the flow of information to the cortex. Philos Trans R Soc Lond B Biol Sci. 2002; 357:1695-1708. DOI: 10.1098/rstb.2002.1161 [PubMed: 12626004] 
48. Oh SW, et al. A mesoscale connectome of the mouse brain. Nature. 2014; 508:207-214. DOI: 10.1038/nature13186 [PubMed: 24695228]

49. Hunnicutt BJ, et al. A comprehensive thalamocortical projection map at the mesoscopic level. Nat Neurosci. 2014; 17:1276-1285. [PubMed: 25086607]

50. Bolkan SS, Carvalho Poyraz F, Kellendonk C. Using human brain imaging studies as a guide toward animal models of schizophrenia. Neuroscience. 2016; 3:77-98.

51. Johansson JD. Spectroscopic method for determination of the absorption coefficient in brain tissue. J Biomed Opt. 2010; 15:057005. [PubMed: 21054121]

52. Mattis J, et al. Principles for applying optogenetic tools derived from direct comparative analysis of microbial opsins. Nat Methods. 2012; 9:159-172.

53. Paxinos, G., Franklin, KBJ. The Mouse Brain in Stereotaxic Coordinates. Academic Press; 2001.

54. Delevich K, Tucciarone J, Huang ZJ, Li B. The mediodorsal thalamus drives feedforward inhibition in the anterior cingulate cortex via parvalbumin interneurons. J Neurosci. 2015; 35:5743-5753. DOI: 10.1523/JNEUROSCI.4565-14.2015 [PubMed: 25855185]

55. Vinck M, van Wingerden M, Womelsdorf T, Fries P, Pennartz CM. The pairwise phase consistency: a bias-free measure of rhythmic neuronal synchronization. Neuroimage. 2010; 51:112-122. DOI: 10.1016/j.neuroimage.2010.01.073 [PubMed: 20114076] 

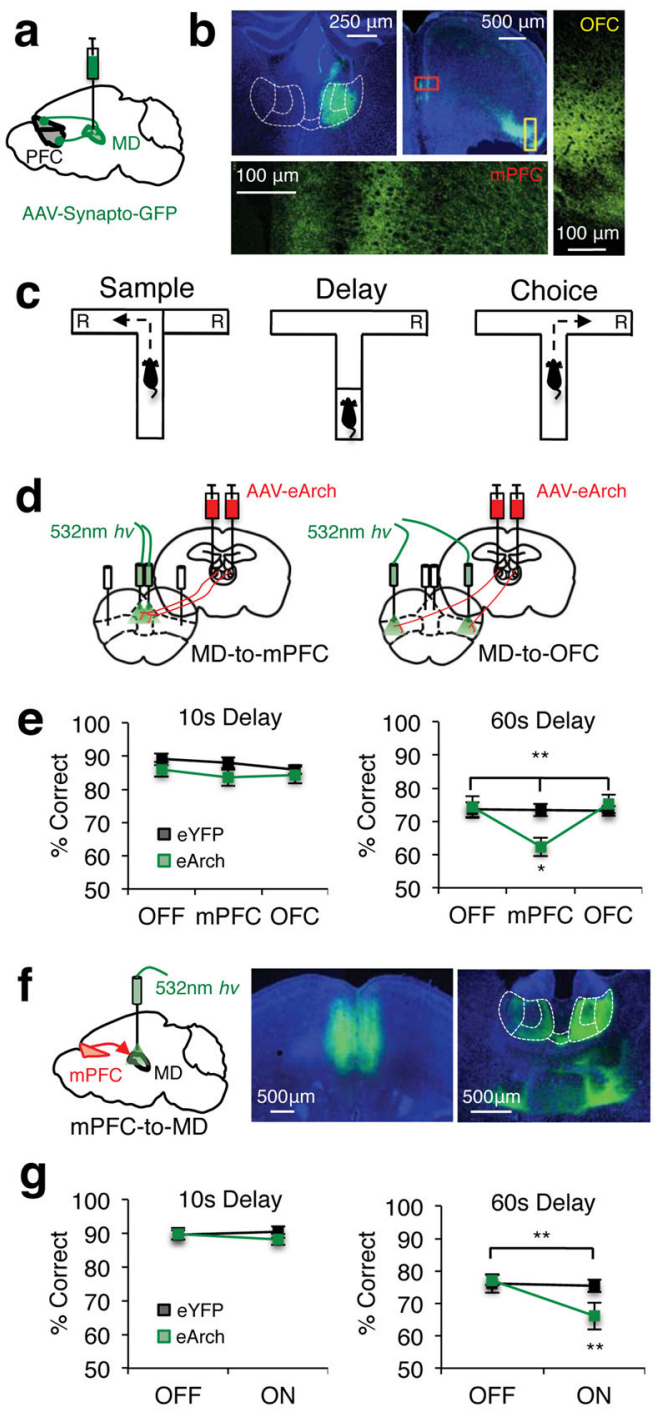

Figure 1. Reciprocal MD-mPFC activity is required for spatial working memory (a) Schema of viral delivery of AAV1-CBA-Synaptophysin-GFP for visualization of MD-toPFC synaptic contacts. (b) Top left: Representative expression of synaptophysin-GFP in MD cell bodies. Top Middle: Synaptophysin-GFP+ MD terminals in PFC. Bottom/Right: Confocal images of synaptophysin-GFP+ MD terminals in medial prefrontal (bottom; mPFC) and dorsolateral orbitofrontal cortex (right; OFC). (c) Schema of a single trial of the DNMS T-maze. "R" indicates reward locations. (d) Schema of viral delivery of AAV5-hSyneArch3.0-eYFP to MD and illumination of MD-to-mPFC (left) or MD-to-OFC (right) terminals within single animals. (e) Percent correct performance in the DNMS T-maze at 10s (left) or 60s (right) delays in eYFP (black trace) and eArch-expressing (green trace) mice (eYFP $n=13$; eArch $n=12$; 10s data: 2-tailed, rmANOVA light $x$ group, $p=0.67$; 60s data: 2tailed, rmANOVA light $\mathrm{x}$ group, $* * \mathrm{p}=0.003, \mathrm{~F}(2,46)=6.73 ;$-tailed, paired t-test eArch OFF vs. mPFC, $\left.{ }^{*} \mathrm{p}=0.02, \mathrm{t}(11)=-2.74\right)$. (f) Left: Schema of viral delivery of eArch-eYFP or eYFP to the mPFC and illumination of mPFC-to-MD terminals. Middle: Representative viral expression in mPFC cell bodies. Right: mPFC terminals projecting to the MD (outlined 
in white and parceled by lateral, central and medial subnuclei). (g) As in e but for mice receiving $\mathrm{mPFC}$-to-MD terminal illumination (eYFP $\mathrm{n}=13$; eArch $\mathrm{n}=14 ; 10$ s data: 2-tailed, rmANOVA light $\mathrm{x}$ group, $\mathrm{p}=0.35 ; 60$ s data: 2 -tailed, rmANOVA light $\mathrm{x}$ group, ${ }^{* *} \mathrm{p}=0.002$, $\mathrm{F}(1,25)=12.1$; 2-tailed, paired t-test eArch light $\mathrm{ON}$ vs. $\mathrm{OFF}, * * \mathrm{p}=0.001, \mathrm{t}(13)=4.19)$. Error bars depict SEM throughout. 


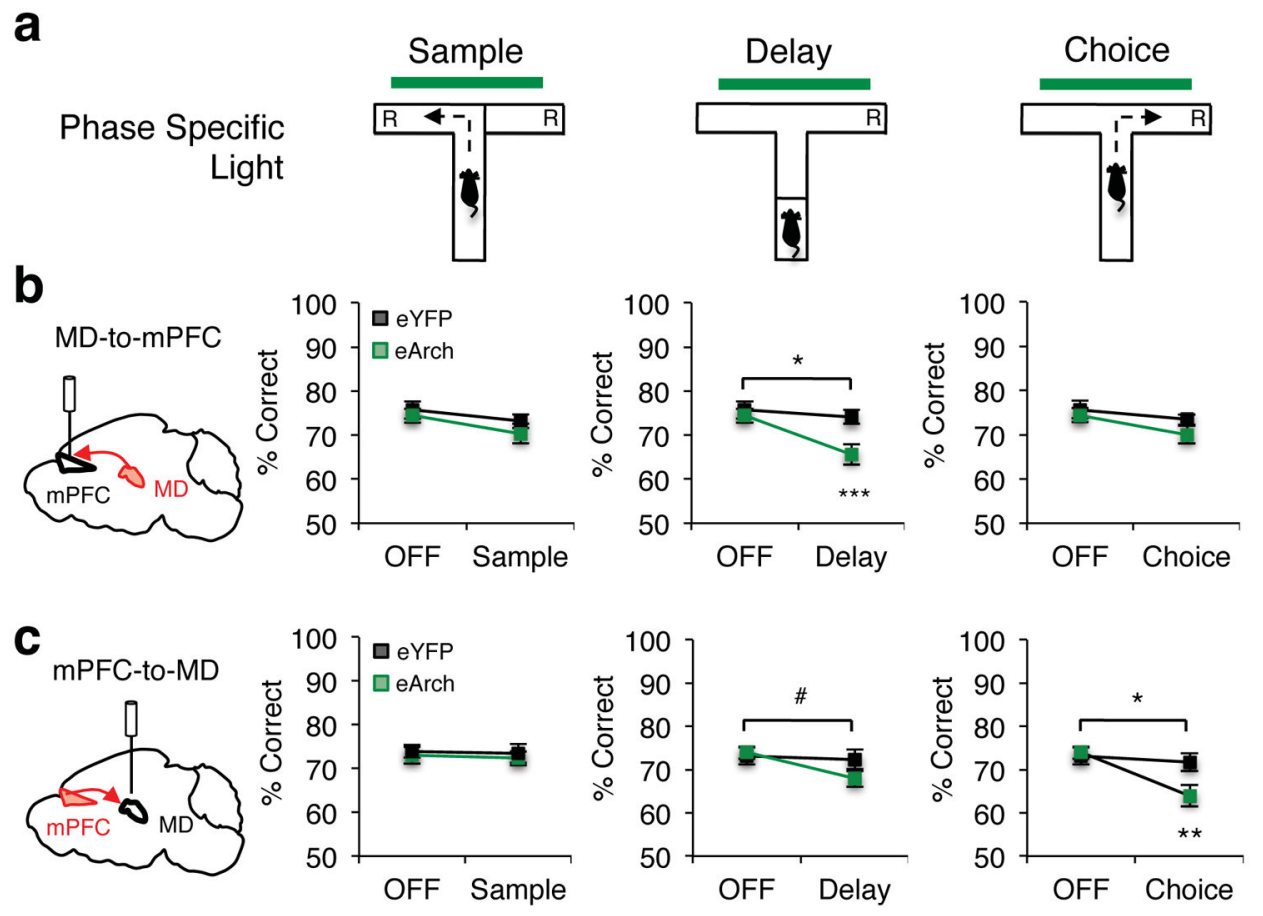

Figure 2. Discrete task phases depend on distinct MD-mPFC interactions

(a) Schema of terminal illumination restricted to the sample, delay or choice phase of the DNMS T-maze. (b) Percent correct performance in mice receiving MD-to-mPFC terminal illumination (eYFP, $\mathrm{n}=11$, black, eArch, $\mathrm{n}=17$, green) during the sample phase (rmANOVA light $\mathrm{x}$ group, $\mathrm{p}=0.59$ ), the delay phase (2-tailed rmANOVA light $\mathrm{x}$ group, ${ }^{*} \mathrm{p}=0.016$, $\mathrm{F}(1,26)=6.7$; 2-tailed, paired t-test eArch light OFF vs. ON Delay, ***p=0.0003, $\mathrm{t}(16)=4.57)$ or choice phase (2-tailed rmANOVA light x group, $\mathrm{p}=0.51$ ). (c) DNMS T-maze performance for mice receiving mPFC-to-MD terminal illumination (eYFP, $\mathrm{n}=13$, black, eArch, $\mathrm{n}=14$, green) during the sample phase (2-tailed rmANOVA light $\mathrm{x}$ group, $\mathrm{p}=0.94$ ), delay phase (2tailed rmANOVA light $x$ group, $\# \mathrm{p}=0.073, \mathrm{~F}(1,25)=3.51$ ) or choice phase (rmANOVA light $\mathrm{x}$ group, ${ }^{*} \mathrm{p}=0.02, \mathrm{~F}(1,25)=6.62 ; 2$-tailed, paired t-test eArch light OFF vs. ON Choice, $* * \mathrm{p}=0.002, \mathrm{t}(13)=3.85)$. Error bars depict SEM throughout. 

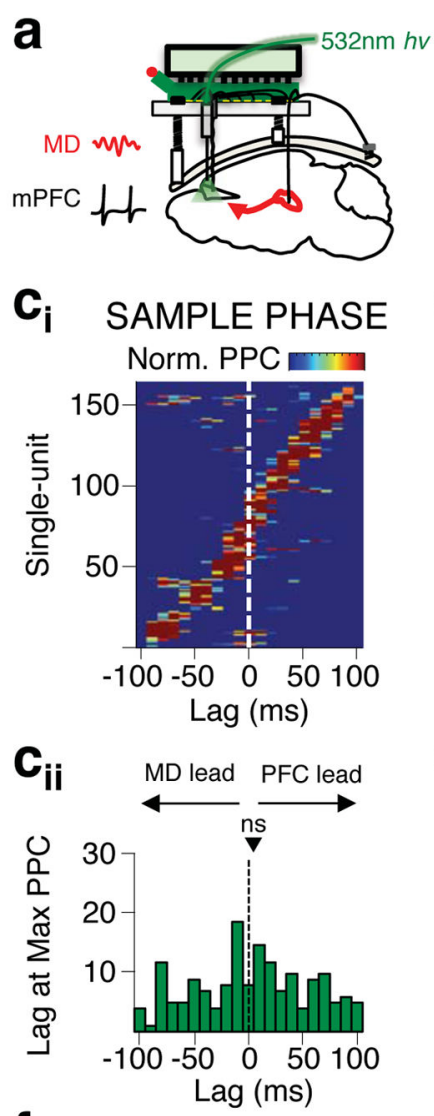

f

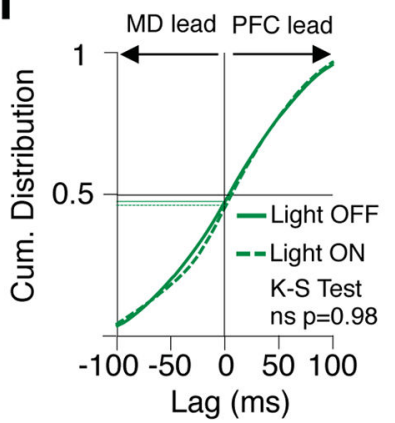

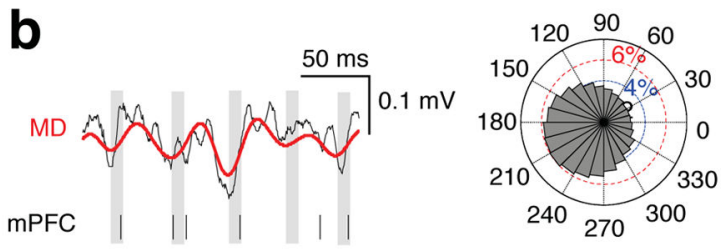
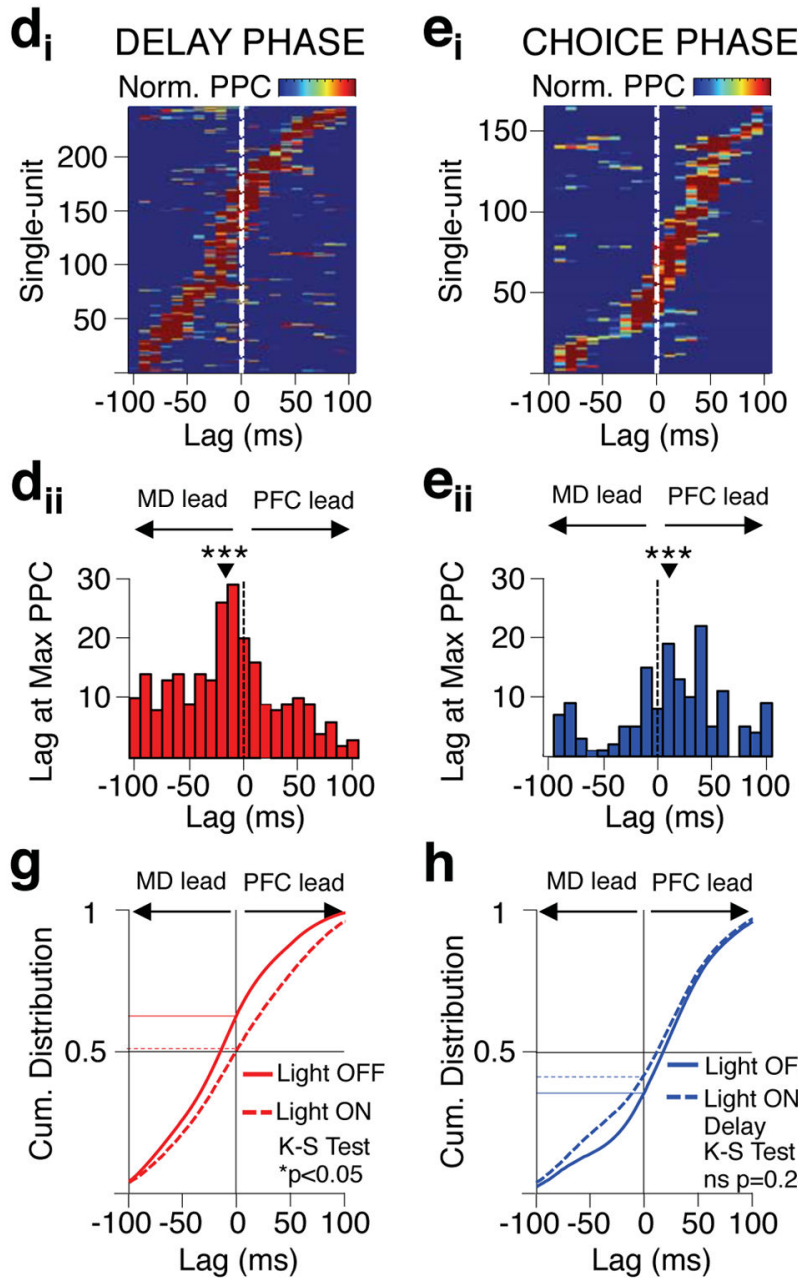

Figure 3. MD-mPFC functional directionality dynamically shifts across task phases (a) Schema of simultaneous recording of MD LFP and mPFC single-units. (b) Representative mPFC single-unit phase-locked to MD LFP filtered in the beta frequency range $(13-30 \mathrm{~Hz})$. Left: Red line depicts filtered beta oscillation overlaid on raw MD LFP (black line). Vertical black lines below indicate simultaneous mPFC spike times with grey shading displaying the trough of the simultaneously recorded MD beta oscillation. Right: Polar plot of the distribution of mPFC spikes relative to a single cycle of the MD beta oscillation for the same unit. $\left(\mathbf{c}_{\mathbf{i}}\right)$ Normalized phase-locking values (pairwise phase comparison (PPC)) for each mPFC neuron during the sample phase of the DNMS T-maze after shifting mPFC spikes in $10 \mathrm{~ms}$ steps $+/-100 \mathrm{~ms}$. Only mPFC units with peak PPC values meeting Bonferonni-corrected $p$ values are included (Rayleigh's circular test, $\mathrm{p}<0.05 / 21$ ) $(165 / 547)$. $\left(\mathbf{c}_{\mathbf{i i}}\right)$ Histogram displaying the lag at which the peak PPC value for each neuron in 
$\left(\mathbf{c}_{\mathrm{ii}}\right)$ occurred. Black triangle indicates mean lag value across the population (mean $=3.5 \mathrm{~ms}$; 2-tailed Signrank, ns: $\mathrm{p}=0.38 ; \mathrm{z}(164)=0.88)$. $\left(\mathbf{d}_{\mathbf{i}-\mathbf{i i}}\right)$ as in $\mathbf{c}_{\mathbf{i}-\mathbf{i i}}$ but for significantly phaselocked units in the delay phase (246/547; mean $=-14.8 ; 2$-tailed Signrank, ${ }^{* * *} \mathrm{p}=0.000005$; $\mathrm{z}(245)=-4.58) .\left(\mathbf{e}_{\mathbf{i}-\mathbf{i i}}\right)$ as in $\mathbf{c}_{\mathbf{i}-\mathbf{i i}}$ but for significantly phase-locked units in the choice phase (153/547; mean=13.3; 2-tailed Signrank, *** $=0.0002 ; \mathrm{z}(152)=3.68)$. (f) Cumulative distribution of significantly phase-locked units during the sample phase across lag times. Solid green curve indicates light off trials and dotted green curve indicates light on sample trials (2-sample Kolmogorov-Smirnov; $\mathrm{ns}, \mathrm{p}=0.98 ; \mathrm{k}=0.049$ ). Horizontal black line indicates $50 \%$ proportion, while vertical black line indicates lag time of 0 . (g) As in $\mathbf{f}$ but for significantly phase-locked units during the delay phase on light off trials (solid red curve) and light on delay trials (dotted red curve) (2-sample Kolmogorov-Smirnov; * $\mathrm{p}=0.01$; $\mathrm{k}=0.14$ ). (h) As in $\mathbf{f}$ but for significantly phase-locked units during the choice phase on light off trials (solid blue curve) and light on delay trials (dotted blue curve) (2-sample Kolmogorov-Smirnov; $\mathrm{ns}, \mathrm{p}=0.28 ; \mathrm{k}=0.11$ ). 
a

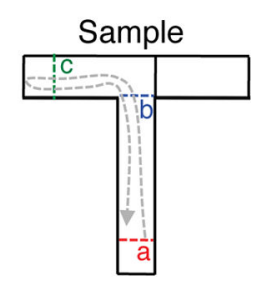

b
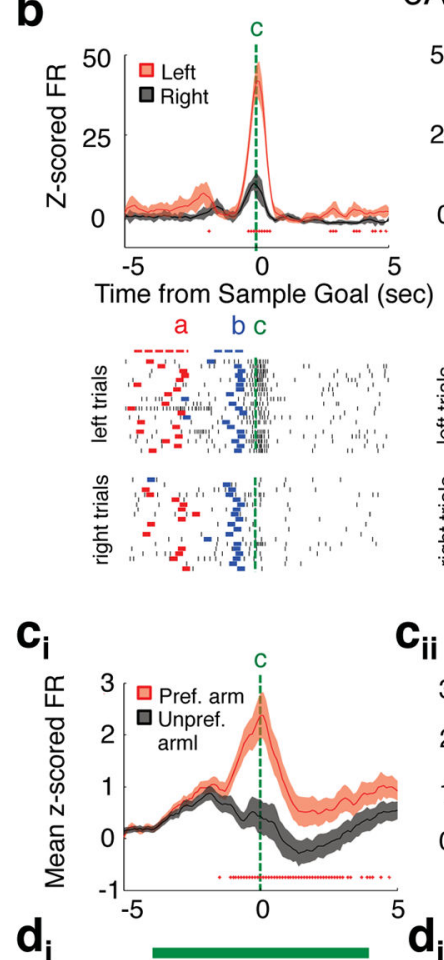

$\mathbf{d}_{\mathrm{i}}$

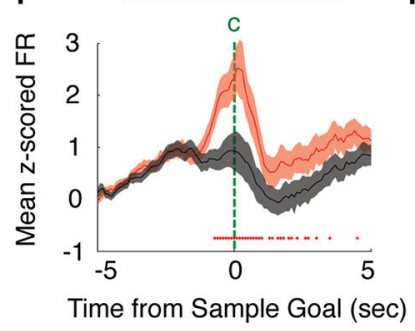

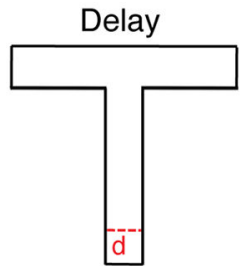

eArch Example Single-unit

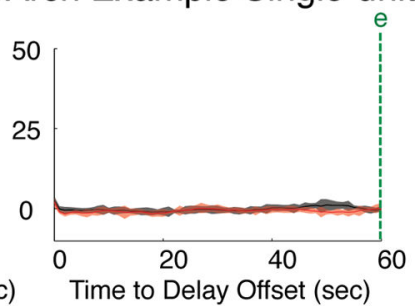

ef
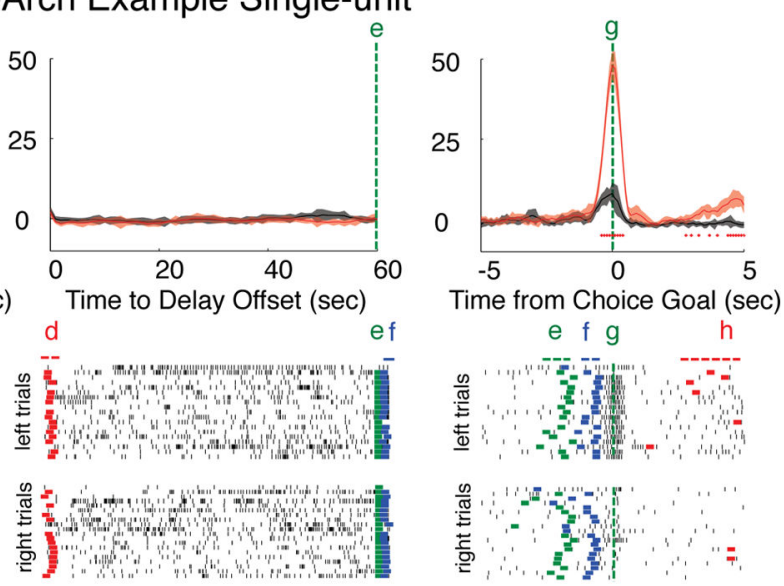

$\mathbf{c}_{\mathrm{ii}}$ All eArch Single-units

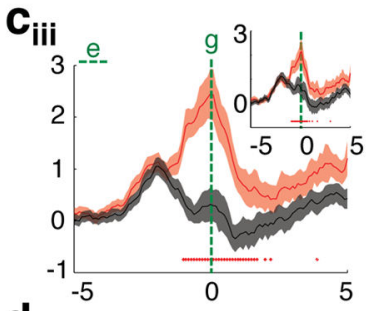

$\mathbf{d}_{\mathrm{ii}}$
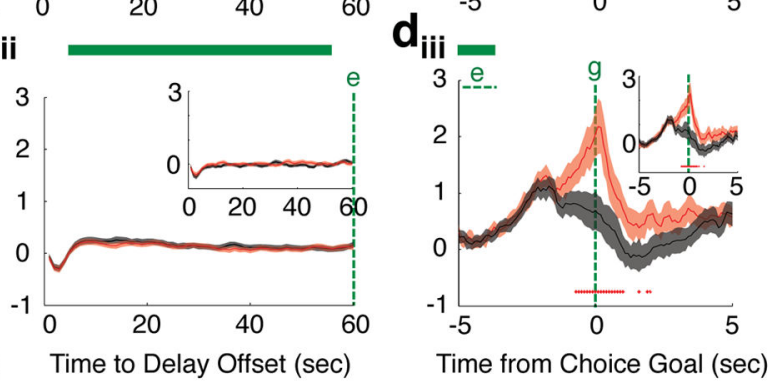

Figure 4. mPFC spatial-tuning is absent during the delay phase and independent of MD input (a) Schema of behavior timestamps for spike alignment on a single DNMS T-maze trial. (b) Example mPFC single-unit spatially-tuned to left arm runs. Top: Peri-event normalized spike rates on left arm (red trace) or right arm (black trace) trials (top; 100ms bins for sample and choice, $1 \mathrm{~s}$ bins for delay). Bottom: Raster plots of raw spike times on left and right trials. Colored lines in raster plots display trial $\mathrm{x}$ trial event timestamps indicated in a above. (c) Normalized firing rate on light off preferred arm trials (red trace) or light off unprefered arm trials (black trace) averaged across all eArch single-units (891 units from 9 eArch mice). Arm preference was determined from firing rate differences on sample arm runs $\left(\mathbf{c}_{\mathbf{i}-\mathrm{iii}}\right)$ or choice arm runs $\left(\mathbf{c}_{\mathrm{ii}-\mathrm{iii}}\right.$, insets). $(\mathbf{d})$ As in $\mathbf{c}$, but for trials in which MD-to- 
mPFC terminals were inhibited during the sample $\left(\mathbf{d}_{\mathbf{i}}\right)$ or delay $\left(\mathbf{d}_{\mathbf{i i}}\right)$ phases. In all normalized firing rate plots, red asterisks indicate bins with 2-tailed Wilcoxon sign-rank (population comparison) or 2-tailed Wilcoxon rank-sum (single-unit comparison) significance at Bonferroni-corrected $p$ values $(\mathrm{p}<0.0005$ sample and choice; $\mathrm{p}<0.00083$ delay). Error bars depict SEM throughout. 

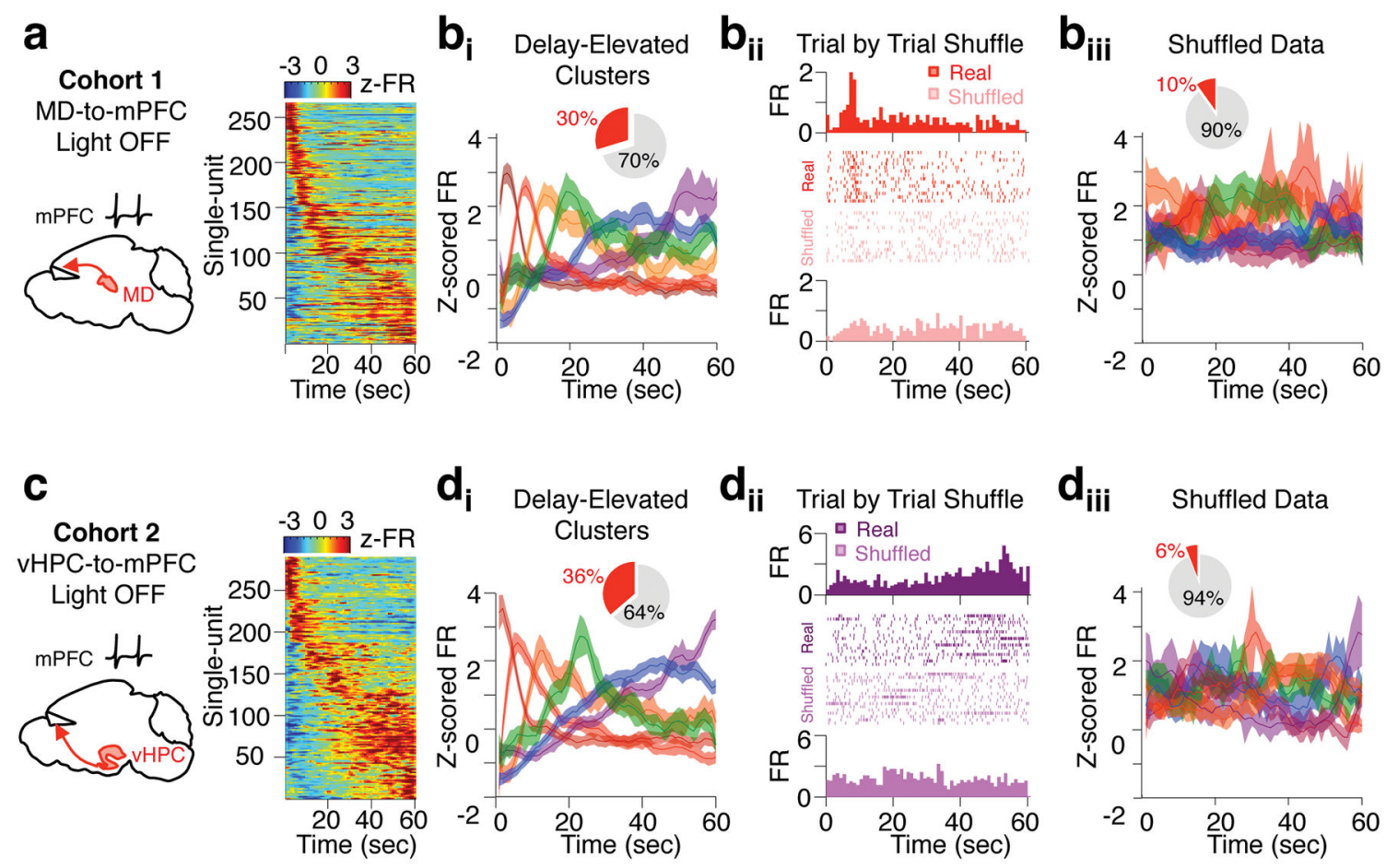

Figure 5. Delay-elevated mPFC neurons exhibit temporally sparse and sequential activity that tiles the delay phase

(a) Normalized firing rates during the delay phase of the DNMS T-maze in a subpopulation of mPFC single-units that exhibit significant elevations in delay period activity $(266 / 891$ units from a cohort of 9 mice expressing eArch in the MD). Normalized firing rates were averaged across all light off trials. Single-units were then sorted by time of peak firing rate. ( $\left.\mathbf{b}_{\mathbf{i}}\right)$ Mean z-scored firing rate of delay-elevated units identified in a after clustering into six groups based on temporal correlation in firing rates. Inset: Proportion of all mPFC neurons

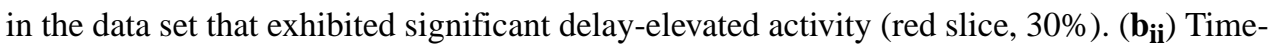
triggered histogram and trial-by-trial raster plot of an example delay-elevated mPFC unit. Histograms and rasters of raw spikes from real data (top) and shuffled versions of the data (bottom) are shown. ( $\left.\mathbf{b}_{\mathbf{i i i}}\right)$ Delay-elevated neurons identified as in $\mathbf{a}$ and clustered as in $\mathbf{b}_{\mathbf{i}}$ but from a trial-by-trial shuffled version of the entire data set. Inset: Proportion of all mPFC neurons in the shuffled data set that exhibit significant delay-elevated activity according to criterion used in $\mathbf{a}(88 / 891,10 \%)$. (c,d) As in $\mathbf{a}$ and $\mathbf{b}_{\mathbf{i}}$ but for mPFC units obtained from an

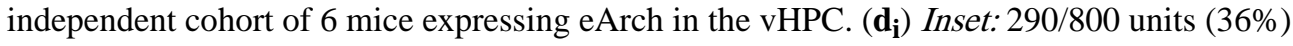
exhibit delay-elevated activity according to criterion in a. (d $\mathbf{d}_{\text {iii }}$ Inset: 47/800 units exhibit delay-elevated activity following trial-by-trial shuffling of the entire data set as in $\mathbf{b}_{\mathbf{i i}}$. Example single units are colored according to their clustered group in $\mathbf{b}_{\mathbf{i}}$ and $\mathbf{d}_{\mathbf{i}}$, respectively. 

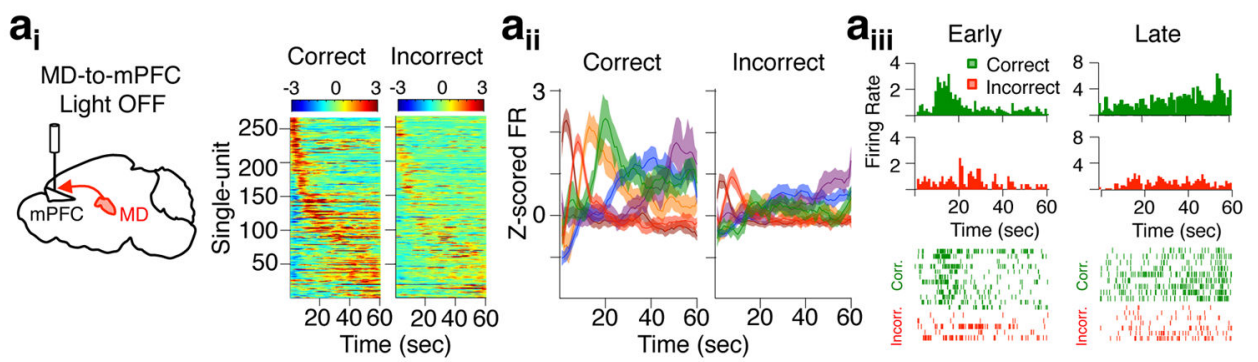

$\mathbf{b}_{\mathbf{i}}$
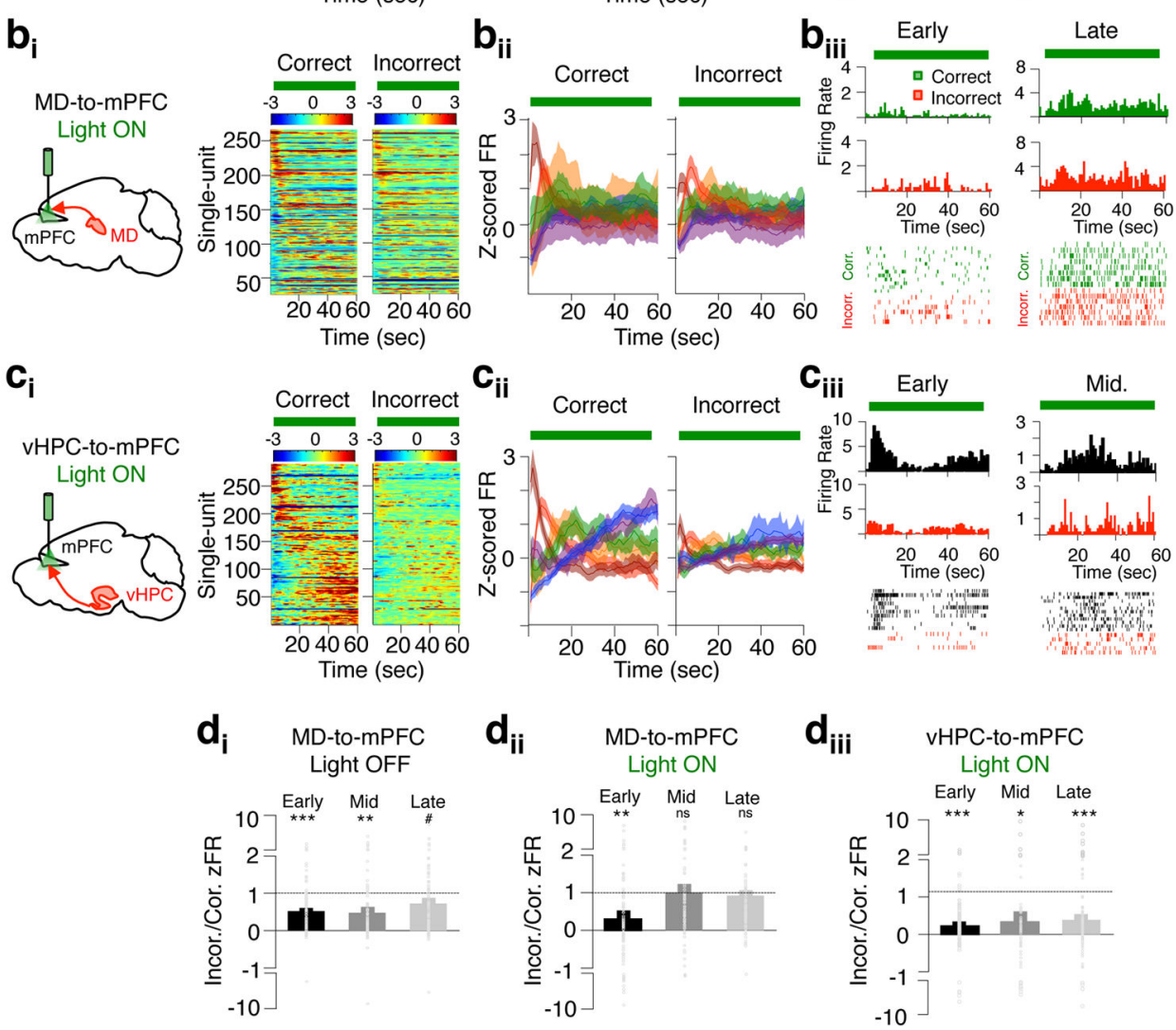

Figure 6. Delay-elevated mPFC activity is diminished on incorrect trials and selectively depends on MD inputs

$\left(\mathbf{a}_{\mathbf{i}}\right)$ Normalized firing rates of delay-elevated $\mathrm{mPFC}$ neurons during light off trials, and parsed by correct or incorrect behavioral performance (266/8919 units from mice expressing eArch in the MD). ( $\left.\mathbf{a}_{\mathbf{i i}}\right)$ Mean normalized firing rate of delay-elevated units after clustering into six groups based on temporal correlations in firing rates. $\left(\mathbf{a}_{\text {iii }}\right)$ Time-triggered histograms and trial-by-trial raster plots from representative delay-elevated units exhibiting early (left) or late (right) delay peaks. Only spikes from light off trials are included, and are plotted separately for correct (green) or incorrect (red) trials. ( $\mathbf{b}_{\mathbf{i}-\mathrm{iii}}$ ) as in $\mathbf{a}_{\mathbf{i}-\mathrm{iii}}$ but for trials in which MD-to-mPFC inputs were inhibited during the delay phase. The same single-units shown in the light off condition in $\mathbf{a}_{\mathrm{iii}}$ are shown in the MD-to-mPFC light on delay condition in $\mathbf{b}_{\mathbf{i i i}}$. $\left(\mathbf{c}_{\mathbf{i}-\text { iii }}\right)$ As in $\mathbf{a}_{\mathbf{i} \text {-iii }}$ but for delay-elevated mPFC units obtained from 6 mice expressing eArch in the vHPC (290/800). Only trials in which vHPC-to-mPFC inputs were inhibited during the delay are included. ( $\left.\mathbf{d}_{\mathbf{i}}\right)$ Ratio of correct/incorrect normalized firing at time of peak firing on all light off trials, averaged across units grouped by early (91), middle 
(83) or late (92) peak times. Groupings reflect the first two, middle two or last two clusters in $\mathbf{a}_{\mathbf{i i}}$. Overlaid circles display all individual single-units. Significance was determined using a 2-tailed t-test against a distribution with mean of $1(* * * \mathrm{p}<0.001, \mathrm{t}(90)=-5.65$;

$* * \mathrm{p}=0.0015, \mathrm{t}(82)=-3.29 ; \# \mathrm{p}=0.07, \mathrm{t}(91)=-1.82) .\left(\mathbf{d}_{\mathbf{i i}}\right)$ As in $\left(\mathbf{d}_{\mathbf{i}}\right)$ but for MD-to-mPFC light on delay trials only $\left({ }^{* *} \mathrm{p}=0.003, \mathrm{t}(90)=-3.07\right.$; not significant $\left.(\mathrm{ns})\right)$. $\left(\mathbf{d}_{\mathrm{iii}}\right)$ As in $\mathbf{d}_{\mathbf{i}}$ but for vHPC-to-mPFC light on delay trials only $(* * * \mathrm{p}<0.001, \mathrm{t}(102)=-6.24 ; * \mathrm{p}=0.018$, $\mathrm{t}(64)=2.42 ; * * * \mathrm{p}=0.0001, \mathrm{t}(121)=-3.97)$. Error bars depict SEM throughout. 

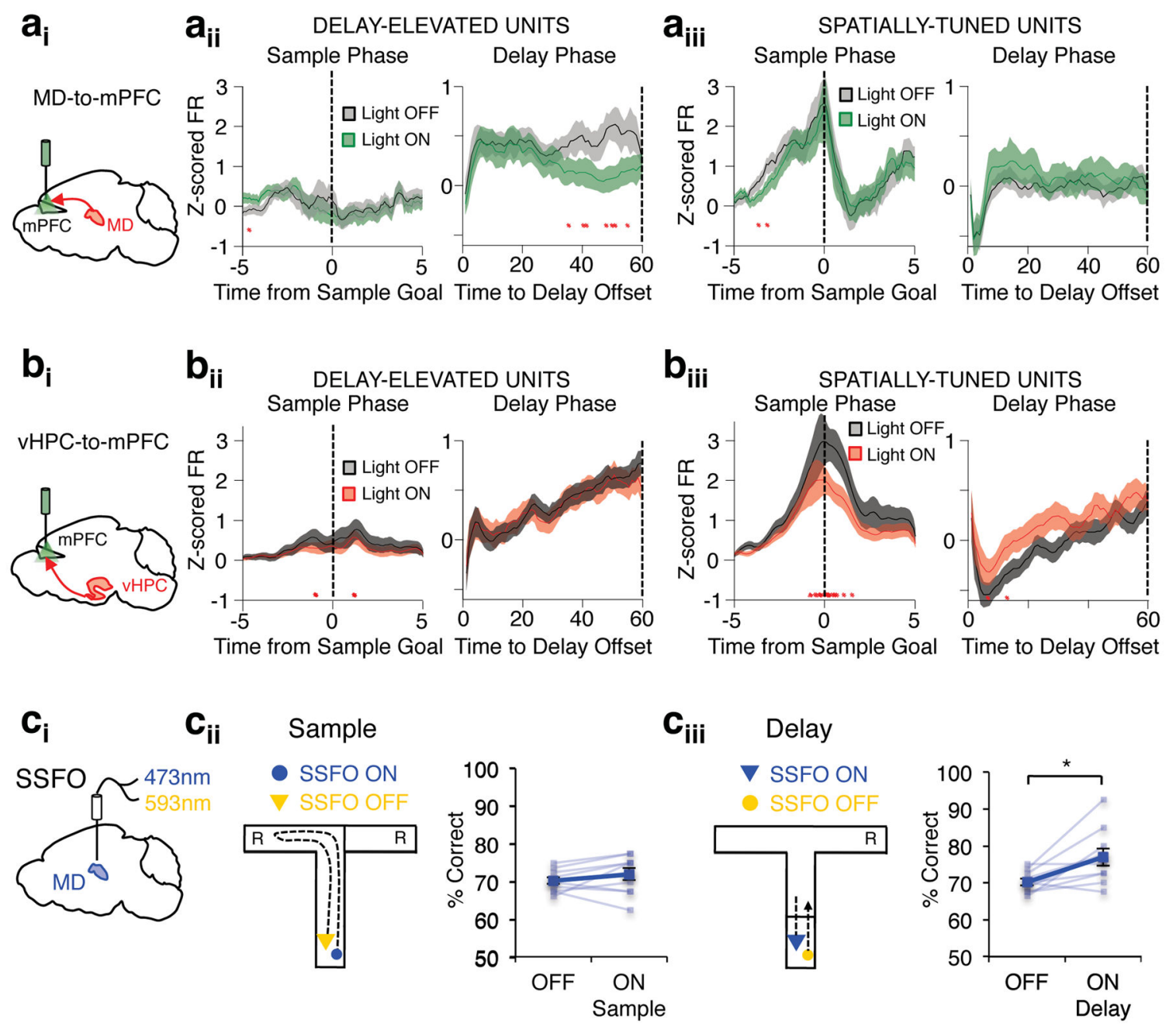

Figure 7. MD activity sustains mPFC delay activity in an input and task phase specific manner (a) Normalized firing rates in delay-elevated (266/891) ( $\left.\mathbf{a}_{\mathbf{i i}}\right)$ and spatially-tuned (250/891) (a $\left.\mathbf{a}_{\text {iii }}\right)$ mPFC neurons obtained from 9 mice expressing eArch in the MD and receiving taskphase specific MD-to-mPFC inhibition during either the sample or delay phases of the DNMS T-maze. Only correct trials are included, which are parsed by light off (black trace) or light on (green trace) conditions. Red asterisks denote bins with Wilcoxon sign-rank significance ( $\mathrm{p}<0.0005$ sample and choice; $\mathrm{p}<0.00083$ delay). (b) As in a but for $\mathrm{mPFC}$ neurons obtained from 6 mice expressing eArch in the vHPC (800 units) and receiving taskphase specific vHPC-to-mPFC inhibition. Delay-elevated: 290/800. Spatially-tuned: 250/800. ( $\mathbf{c}_{\mathbf{i}}$ ) Schema of stabilized step function opsin (SSFO, hChr2(C128S/D156A) activation and deactivation of MD activity. $\left(\mathbf{c}_{\mathbf{i i}}\right)$ Schema of SSFO activation at sample phase onset and deactivation at sample phase offset (left). Percent correct performance in the DNMS T-maze in 9 SSFO-expressing mice during light off and on sample trials (right). Transparent blue lines reveal individual mouse performance, while thick blue line indicates group mean performance (2-tailed, paired t-test: $\mathrm{p}=0.26 ; \mathrm{t}(8)=-1.22)$. $\left(\mathbf{c}_{\mathrm{iii}}\right)$ As in $\mathbf{c}_{\mathrm{ii}}$ but for mice receiving SSFO activation of the MD at delay onset and deactivation at delay offset (2- 
tailed rmANOVA on all trial types, Light effect: $\mathrm{p}=\mathrm{F}(7)=7.75$, $\mathrm{p}<0.01$; 2-tailed, paired t-test, light off vs. on delay: $\mathrm{p}=0.014, \mathrm{t}(8)=-3.14)$. Error bars depict SEM throughout. 